\title{
Sensitivity of T2KK to the non-standard interaction in propagation
}

\author{
Haruna Oki* and Osamu Yasuda \\ Department of Physics, Tokyo Metropolitan University, \\ Minami-Osawa, Hachioji, Tokyo 192-0397, Japan
}

(Dated: October 12, 2018)

\begin{abstract}
Assuming only the non-zero electron and tau neutrino components $\epsilon_{e e}, \epsilon_{e \tau}, \epsilon_{\tau \tau}$ of the nonstandard matter effect and postulating the atmospheric neutrino constraint $\epsilon_{\tau \tau}=\left|\epsilon_{e \tau}\right|^{2} /\left(1+\epsilon_{e e}\right)$, we study the sensitivity to the non-standard interaction in neutrino propagation of the T2KK neutrino long-baseline experiment. It is shown that T2KK can constrain the parameters $\left|\epsilon_{e e}\right| \lesssim 1$, $\left|\epsilon_{e \tau}\right| \lesssim 0.2$. It is also shown that if $\left|\epsilon_{e \tau}\right|$ and $\theta_{13}$ are large, then T2KK can determine the Dirac phase and the phase of $\epsilon_{e \tau}$ separately, due to the information at the two baselines. We also provide an argument that the components $\left|\epsilon_{\alpha \mu}\right|(\alpha=e, \mu, \tau)$ must be small for the disappearance oscillation probability to be consistent with high-energy atmospheric neutrino data, which justifies our premise that these quantities are negligible.
\end{abstract}

PACS numbers: 14.60.Pq, 14.60.St

* Present address: Toppan Printing Co., Ltd., Japan.

$\dagger$ yasuda:at phys.metro-u.ac.jp 


\section{INTRODUCTION}

It has been shown by experiments with solar and atmospheric neutrinos [1] that neutrinos have masses and mixings. In the standard three-flavor framework, neutrino oscillations are described by three mixing angles, $\theta_{12}, \theta_{13}, \theta_{23}$, one CP phase $\delta$, and two independent mass-squared differences, $\Delta m_{21}^{2}$ and $\Delta m_{31}^{2}$. The values of the set of the parameters $\left(\Delta m_{21}^{2}\right.$, $\left.\theta_{12}\right)$ and $\left(\Delta m_{31}^{2}, \theta_{23}\right)$ have been determined to a certain precision by solar and atmospheric neutrino experiments, respectively. On the other hand, only the upper bound on $\theta_{13}$ is known 1 , $\sin ^{2} \theta_{13}<0.04$ at $90 \% \mathrm{CL}$, and there is an absence of information on $\delta$. In future neutrino long-baseline experiments (see, e.g., Ref. [8]), the values of $\theta_{13}$ and $\delta$ are expected to be determined precisely. As in the case of B factories [9, 10], such highly precise measurements will enable us to search for deviation from the standard three-flavor oscillations. One such possibility, which will be discussed in this paper, is the effective non-standard neutral current-neutrino interaction with matter [11 13], given by

$$
\mathcal{L}_{\text {eff }}^{\mathrm{NSI}}=-2 \sqrt{2} \epsilon_{\alpha \beta}^{f P} G_{F}\left(\bar{\nu}_{\alpha} \gamma_{\mu} P_{L} \nu_{\beta}\right)\left(\bar{f} \gamma^{\mu} P f^{\prime}\right),
$$

where $f$ and $f^{\prime}$ stand for fermions (the only relevant ones are electrons, $\mathrm{u}$, and d quarks), $G_{F}$ is the Fermi coupling constant, and $P$ stands for a projection operator that is either $P_{L} \equiv\left(1-\gamma_{5}\right) / 2$ or $P_{R} \equiv\left(1+\gamma_{5}\right) / 2$. In the presence of the interaction Eq. (11), the standard matter effect [11, 14] is modified. Since we discuss the long-baseline experiments on the Earth, we make an approximation that the number densities of electrons $\left(N_{e}\right)$, protons, and neutrons are equal 2 By introducing the notation $\epsilon_{\alpha \beta} \equiv \sum_{P}\left(\epsilon_{\alpha \beta}^{e P}+3 \epsilon_{\alpha \beta}^{u P}+3 \epsilon_{\alpha \beta}^{d P}\right)$, the hermitian $3 \times 3$ matrix of the matter potential becomes

$$
\mathcal{A} \equiv A\left(\begin{array}{ccc}
1+\epsilon_{e e} & \epsilon_{e \mu} & \epsilon_{e \tau} \\
\epsilon_{\mu e} & \epsilon_{\mu \mu} & \epsilon_{\mu \tau} \\
\epsilon_{\tau e} & \epsilon_{\tau \mu} & \epsilon_{\tau \tau}
\end{array}\right)
$$

where $A \equiv \sqrt{2} G_{F} N_{e}$.

Constraints on $\epsilon_{\alpha \beta}$ from various neutrino experiments have been discussed in Refs. [1523]. Since the coefficients $\epsilon_{\alpha \beta}$ in Eq. (2) are given by $\epsilon_{\alpha \beta} \sim \epsilon_{\alpha \beta}^{e}+3 \epsilon_{\alpha \beta}^{u}+3 \epsilon_{\alpha \beta}^{d}$, considering the constraints by Refs. [15-23], we have the following constraints [24] at 90\% CL 3 :

$$
\left(\begin{array}{rlrl}
\left|\epsilon_{e e}\right|<4 \times 10^{0} & \left|\epsilon_{e \mu}\right|<3 \times 10^{-1} & \left|\epsilon_{e \tau}\right|<3 \times 10^{0} \\
& \left|\epsilon_{\mu \mu}\right|<7 \times 10^{-2} & \left|\epsilon_{\mu \tau}\right|<3 \times 10^{-1} \\
& \left|\epsilon_{\tau \tau}\right|<2 \times 10^{1}
\end{array}\right) .
$$

From this prior study, it is known that the bounds on $\epsilon_{e e}, \epsilon_{e \tau}$ and $\epsilon_{\tau \tau}$ are much weaker than $\epsilon_{\alpha \mu}(\alpha=e, \mu, \tau)$, and typically $\epsilon_{\alpha \beta} \sim \mathcal{O}(1)(\alpha, \beta=e, \tau)$ is allowed.

On the other hand, the new physics with components $\epsilon_{\alpha \beta}(\alpha, \beta=e, \tau)$ should be consistent with the high-energy atmospheric neutrino data, which suggest the behavior of the

1 In Refs. 2 [7], a global analysis of the neutrino oscillation data has been performed, in which a nonvanishing best-fit value for $\theta_{13}$ is obtained. This result, however, is compatible with $\theta_{13}=0$ at less than $2 \sigma$, and it is not yet statistically significant enough to be taken seriously.

2 This assumption is not valid in other environments, e.g., in the Sun.

3 Here, we adopt the conservative bounds on $\epsilon_{\alpha \beta}$ which were derived without using the one-loop arguments, because the bounds obtained by the loop-contributions are known to be model-dependent [25]. However, if we accept results based on one-loop arguments, then we get the following bounds at 90\%CL: $\left|\epsilon_{e \mu}\right|<$ $\left[\sum_{P}\left(\left|\epsilon_{e \mu}^{e P}\right|^{2}+\left|3 \epsilon_{e \mu}^{u P}\right|^{2}+\left|3 \epsilon_{e \mu}^{d P}\right|^{2}\right)\right]^{1 / 2} \sim 5 \times 10^{-3}\left[26\right.$ 28] , where $\left|\epsilon_{e \mu}^{e P}\right|<5 \times 10^{-4}[15],\left|\epsilon_{e \mu}^{u P}\right|<3.1 \times 10^{-4}[27,28]$ and $\left|\epsilon_{e \mu}^{d P}\right|<3.1 \times 10^{-4}[27,28]$ are used; $\left|\epsilon_{e \tau}\right|<\left[\sum_{P}\left(\left|\epsilon_{e \tau}^{e P}\right|^{2}+\left|3 \epsilon_{e \tau}^{u P}\right|^{2}+\left|3 \epsilon_{e \tau}^{d P}\right|^{2}\right)\right]^{1 / 2} \sim 1.7$ [27, 28], where $\left|\epsilon_{e \tau}^{e P}\right|<0.32$ [27, 28], $\left|\epsilon_{e \tau}^{u P}\right|<0.28$ [27, 28] and $\left|\epsilon_{e \tau}^{d P}\right|<0.28$ [27, 28] are used. 
disappearance oscillation probability

$$
1-P\left(\nu_{\mu} \rightarrow \nu_{\mu}\right) \sim \sin ^{2} 2 \theta_{\mathrm{atm}} \sin ^{2}\left(\frac{\Delta m_{\mathrm{atm}}^{2} L}{4 E}\right) \propto \frac{1}{E^{2}}
$$

where $\sin ^{2} 2 \theta_{\text {atm }}$ and $\Delta m_{\text {atm }}^{2}$ are the oscillation parameters in the two-flavor formalism. Note that the terms of $\mathcal{O}\left(E^{0}\right)$ and $\mathcal{O}\left(E^{-1}\right)$ are absent in Eq. (44). As shown later, the elements $\epsilon_{\alpha \mu}(\alpha=e, \mu, \tau)$ should be small so as to produce no term of $\mathcal{O}\left(E^{0}\right)$ in Eq. (44). Furthermore, absence of terms of $\mathcal{O}\left(E^{-1}\right)$ in Eq. (4) implies

$$
\left|\epsilon_{e \tau}\right|^{2} \simeq \epsilon_{\tau \tau}\left(1+\epsilon_{e e}\right)
$$

as pointed out in Ref. [29, 30]. When Eq. (5) is satisfied, two of the three eigenvalues of the matrix (2) with $\epsilon_{\alpha \mu}=0(\alpha=e, \mu, \tau)$ become zero. Only in this case, one of the frequencies of the three oscillation modes at high energy coincides with the one in the vacuum oscillation, and the disappearance oscillation probability of $\nu_{\mu}$ behaves as in Eq. (4). The effect of the non-standard interaction in propagation for solar neutrinos has also been discussed in Refs. [19, 22, 23, 31, 32], and Refs. [23] and [32] give a constraint $-0.06<\epsilon_{e \tau}^{d V} \sin \theta_{23}<0.41$ (at $90 \% \mathrm{CL}$ ) and $\left|\epsilon_{e \tau}^{d V}\right| \lesssim 0.4$ (at $\Delta \chi^{2}=4$ for 2 d.o.f.), respectively.

The sensitivity of the ongoing long-baseline experiments to the non-standard interaction in propagation has been studied for MINOS [33] in Refs. [34 37], and for OPERA [38] in Refs. 39, 40]. On the other hand, for the sensitivity of future long-baseline experiments, Ref. [41] provided the sensitivity of the T2KK experiment [42, 43], an extension of the T2K neutrino oscillation experiment [44] with a far detector in Korea, in the two-flavor analysis with $\epsilon_{e \alpha}=0(\alpha=e, \mu, \tau)$. The sensitivity of the reactor and super-beam experiments was discussed in Ref. [45], and the sensitivity of neutrino factories [8, 46] has been discussed by many authors [47 [54].

In the present paper we analyze the sensitivity to the parameters $\epsilon_{\alpha \beta}$ of the T2KK experiment, assuming $\epsilon_{\mu \alpha}=0(\alpha=e, \mu, \tau)$ and $\epsilon_{\tau \tau}=\left|\epsilon_{e \tau}\right|^{2} /\left(1+\epsilon_{e e}\right)$. We do not discuss the so-called parameter degeneracy [55-58], since little is known about parameter degeneracy in the presence of the new physics, and the study of the subject is beyond the scope of this paper.

The paper is organized as follows. In sect!, we discuss the constraints on the $\epsilon_{\alpha \beta}$ parameters from the high-energy atmospheric neutrino data. In sect III, we analyze the sensitivity of T2KK to the new physics parameters. In sect.IV, we draw our conclusions. In the appendices $\mathrm{A} G$ we provide details of the derivation of the analytic formulae for the oscillation probabilities and their high-energy behaviors.

\section{ATMOSPHERIC NEUTRINOS AND THE NON-STANDARD INTERACTION IN PROPAGATION}

In Refs. [29, 30], it was pointed out that the new physics should be consistent with the constraint imposed by the atmospheric neutrino data, which suggest that the disappearance oscillation probability at high-energy satisfies Eq. (44). In the standard three-flavor scheme, the high-energy behavior is

$$
\lim _{E \rightarrow \infty} \frac{1-P\left(\nu_{\mu} \rightarrow \nu_{\mu}\right)}{\left(\Delta E_{31} / A\right)^{2}} \simeq \lim _{E \rightarrow \infty} \frac{1}{\left(\Delta E_{31} / A\right)^{2}}\left[4 \frac{\left|U_{\mu 3}\right|^{2}}{c_{13}^{2}}\left(1-\frac{\left|U_{\mu 3}\right|^{2}}{c_{13}^{2}}\right) \sin ^{2}\left(\frac{c_{13}^{2} \Delta E_{31} L}{2}\right)\right.
$$




$$
\begin{array}{r}
\left.+4\left(\frac{\Delta E_{31}}{A}\right)^{2}\left|U_{\mu 3}\right|^{2} s_{13}^{2} \sin ^{2}\left(\frac{A L}{2}\right)\right] \\
\simeq \sin ^{2} 2 \theta_{23}\left(\frac{c_{13}^{2} A L}{2}\right)^{2}+s_{23}^{2} \sin ^{2} 2 \theta_{13} \sin ^{2}\left(\frac{A L}{2}\right),
\end{array}
$$

where $\Delta E_{j k} \equiv E_{j}-E_{k} \simeq \Delta m_{j k}^{2} / 2 E$. (See the appendix $\AA$ for derivation of the oscillation probability in constant density matter and the appendix $B$ for derivation of its high-energy behavior (6)). In the following discussions, we assume the generic matter potential (2), and derive the high-energy behavior

$$
1-P\left(\nu_{\mu} \rightarrow \nu_{\mu}\right) \simeq c_{0}+\frac{c_{1}}{E}+\mathcal{O}\left(\frac{1}{E^{2}}\right),
$$

and postulate $\left|c_{0}\right| \ll 1$ and $\left|c_{1}\right| \ll 1$. Note that the term of $\mathcal{O}\left(E^{-2}\right)$ corresponds to the standard results (44) in the two-flavor scheme or (6) in the three-flavor case, where the information on the atmospheric neutrino oscillation parameters appears. In the presence of the matter potential (2), as we discussed in detail in the appendix C] assuming the constant density of matter, we can obtain the analytic form for the behavior (4) of the disappearance oscillation probability at high energy, in which $\left|A / \Delta E_{31}\right| \equiv \sqrt{2} G_{F} N_{e} /\left(\left|\Delta m_{31}^{2}\right| / 2 E\right) \ll 1$ is satisfied. The leading term $c_{0}$ in Eq. (7) is given by

$$
\begin{aligned}
c_{0} \simeq & {\left[1-\frac{1+\epsilon_{e e}+\epsilon_{\tau \tau}}{\left(1+\epsilon_{e e}\right)\left|\epsilon_{\mu \tau}\right|^{2}+\epsilon_{\tau \tau}\left|\epsilon_{e \mu}\right|^{2}-2 \operatorname{Re}\left(\epsilon_{e \mu} \epsilon_{\mu \tau}\left(\epsilon_{e \tau}\right)^{*}\right)}\left(\frac{\left|\epsilon_{e \mu}\right|^{2}+\left|\epsilon_{\mu \mu}\right|^{2}+\left|\epsilon_{\mu \tau}\right|^{2}}{1+\epsilon_{e e}+\epsilon_{\tau \tau}}-\epsilon_{\mu \mu}\right)^{2}\right] } \\
& \times \sin ^{2}\left[A L \frac{\left(1+\epsilon_{e e}\right)\left|\epsilon_{\mu \tau}\right|^{2}+\epsilon_{\tau \tau}\left|\epsilon_{e \mu}\right|^{2}-2 \operatorname{Re}\left(\epsilon_{e \mu} \epsilon_{\mu \tau}\left(\epsilon_{e \tau}\right)^{*}\right)}{1+\epsilon_{e e}+\epsilon_{\tau \tau}}\right] \\
+ & 4\left[\frac{\left|\epsilon_{e \mu}\right|^{2}+\left|\epsilon_{\mu \mu}\right|^{2}+\left|\epsilon_{\mu \tau}\right|^{2}}{\left(1+\epsilon_{e e}+\epsilon_{\tau \tau}\right)^{2}}-\frac{\left(1+\epsilon_{e e}\right)\left|\epsilon_{\mu \tau}\right|^{2}+\epsilon_{\tau \tau}\left|\epsilon_{e \mu}\right|^{2}-2 \operatorname{Re}\left(\epsilon_{e \mu} \epsilon_{\mu \tau}\left(\epsilon_{e \tau}\right)^{*}\right)}{\left(1+\epsilon_{e e}+\epsilon_{\tau \tau}\right)^{3}}\right] \\
& \times \sin ^{2}\left(\frac{\left(1+\epsilon_{e e}+\epsilon_{\tau \tau}\right) A L}{2}\right) .
\end{aligned}
$$

For Eq. (8) to be consistent with Eq. (4), we should have $\epsilon_{e \mu} \simeq \epsilon_{\mu \mu} \simeq \epsilon_{\mu \tau} \simeq 0$ (see appendix C).

In Refs. [16, 18, 59], the two-flavor analysis of the atmospheric neutrino data with the matter effect

$$
A\left(\begin{array}{cc}
\epsilon_{\mu \mu} & \epsilon_{\mu \tau} \\
\epsilon_{\mu \tau} & \epsilon_{\tau \tau}
\end{array}\right)
$$

was performed. In this case, in the limit $E \rightarrow \infty$ the disappearance oscillation probability results in

$$
1-P\left(\nu_{\mu} \rightarrow \nu_{\mu}\right) \simeq \frac{\left|\epsilon_{\mu \tau}\right|^{2}}{\left(\epsilon_{\mu \mu}-\epsilon_{\tau \tau}\right)^{2} / 4+\left|\epsilon_{\mu \tau}\right|^{2}} \sin ^{2}\left(A L \sqrt{\left(\epsilon_{\mu \mu}-\epsilon_{\tau \tau}\right)^{2} / 4+\left|\epsilon_{\mu \tau}\right|^{2}}\right) .
$$

This suggests that the two parameters $\left|\epsilon_{\mu \tau}\right|$ and $\left|\epsilon_{\mu \mu}-\epsilon_{\tau \tau}\right|$ should be small so as to be consistent with the high-energy behavior (4), and in fact, the authors of Ref. [59] obtained the bounds $\left|\epsilon_{\mu \tau}\right|<1.5 \times 10^{-2}$ and $\left|\epsilon_{\mu \mu}-\epsilon_{\tau \tau}\right|<4.9 \times 10^{-2}$ at $90 \%$ CL. In the three flavor framework, as seen below, $\epsilon_{\alpha \beta} \sim \mathcal{O}(1)(\alpha, \beta=e, \tau)$ can be consistent with the atmospheric 
neutrino data as long as $\epsilon_{\tau \tau}-\left|\epsilon_{e \tau}\right|^{2} /\left(1+\epsilon_{e e}\right) \simeq 0$ is satisfied [29, 30]. We see that the two flavor constraints in Refs. [16, 18, 59] are consistent with three flavor ones as follows: As for $\epsilon_{\tau \tau}$, since $\left|\epsilon_{\mu \mu}\right| \ll 1$, the bound $\left|\epsilon_{\mu \mu}-\epsilon_{\tau \tau}\right| \ll 1$ in the two flavor framework implies $\left|\epsilon_{\tau \tau}\right| \ll 1$. The two flavor framework can be regarded as a subset of the three flavor case in the limiting case $\epsilon_{e e}=\epsilon_{e \tau}=\theta_{13}=\Delta m_{21}^{2}=0$, so the constraint Eq. (5) in the two flavor case leads to $\left|\epsilon_{\tau \tau}\right| \simeq 0$. On the other hand, the bound on $\left|\epsilon_{\mu \tau}\right|$ in the three flavor case is independent of other components $\epsilon_{\alpha \beta}$, so the bound $\left|\epsilon_{\mu \tau}\right| \lesssim \mathcal{O}\left(10^{-2}\right)$ is expected to be valid both in the two and three flavor cases. While $\left|\epsilon_{\mu \mu}\right| \ll 1$ and $\left|\epsilon_{\mu \tau}\right| \ll 1$ were first shown in Refs. [15] and [16], respectively, if we do not accept the one-loop arguments [15] to constrain $\epsilon_{e \mu}$ as in Ref. [25], then the observation in this paper that $\left|\epsilon_{e \mu}\right| \ll 1$ follows from the the atmospheric neutrino constraint is new, although our discussions are based only on an analytical treatment 4

In the following discussions, therefore, let us assume that $\epsilon_{e \mu}=\epsilon_{\mu \mu}=\epsilon_{\mu \tau}=0$. Then we obtain the coefficient $c_{1}$ of the term of the next-to-leading order in $1 / E$ in Eq. (7) (see appendix [D):

$$
c_{1} \simeq-\frac{2 s_{23}^{2} \Delta m_{31}^{2}}{\left(1+\epsilon_{e e}\right) A \Delta t_{32}}\left[\frac{t_{3}^{2}}{\zeta^{\prime}} \sin ^{2}\left\{\frac{\left(1+\epsilon_{e e}\right) \zeta^{\prime} A L}{2 t_{3}}\right\}+\frac{\zeta^{\prime}}{t_{3}^{2}} \sin ^{2}\left\{\frac{\left(1+\epsilon_{e e}\right) t_{3} A L}{2}\right\}\right],
$$

where

$$
\begin{aligned}
\zeta^{\prime} & \equiv \frac{1}{1+\epsilon_{e e}}\left(\epsilon_{\tau \tau}-\frac{\left|\epsilon_{e \tau}\right|^{2}}{1+\epsilon_{e e}}\right) \\
t_{3} & \equiv \frac{1}{2\left(1+\epsilon_{e e}\right)}\left[1+\epsilon_{e e}+\epsilon_{\tau \tau}+\sqrt{\left(1+\epsilon_{e e}+\epsilon_{\tau \tau}\right)^{2}-4\left(1+\epsilon_{e e}\right)^{2} \zeta^{\prime}}\right] \\
\Delta t_{32} & \equiv \frac{1}{2\left(1+\epsilon_{e e}\right)} \sqrt{\left(1+\epsilon_{e e}+\epsilon_{\tau \tau}\right)^{2}-4\left(1+\epsilon_{e e}\right)^{2} \zeta^{\prime}} .
\end{aligned}
$$

Again, for Eq. (9) with Eq. (7) to be consistent with the high-energy behavior (4), we should have

$$
\epsilon_{\tau \tau}-\frac{\left|\epsilon_{e \tau}\right|^{2}}{1+\epsilon_{e e}} \simeq 0
$$

This agrees with the conclusion $\epsilon_{\tau \tau} \simeq\left|\epsilon_{e \tau}\right|^{2} /\left(1+\epsilon_{e e}\right)$ in Refs. [29, 30].

Thus, let us assume $\epsilon_{\tau \tau}-\left|\epsilon_{e \tau}\right|^{2} /\left(1+\epsilon_{e e}\right)=0$. In this case, it is convenient to introduce the new variable

$$
t_{\beta} \equiv \tan \beta \equiv \frac{\left|\epsilon_{e \tau}\right|}{1+\epsilon_{e e}}
$$

Then, we have the following high-energy behavior (see appendix E):

$$
\begin{aligned}
\frac{1-P\left(\nu_{\mu} \rightarrow \nu_{\mu}\right)}{\left(\Delta E_{31} / A\right)^{2}} \simeq & 4 \frac{s_{23}^{2}}{\left(c_{13}^{\prime \prime}\right)^{2}}\left\{1-\frac{s_{23}^{2}}{\left(c_{13}^{\prime \prime}\right)^{2}}\right\}\left\{\frac{\left(c_{13}^{\prime \prime}\right)^{2} A L}{2}\right\}^{2} \\
& +\frac{s_{23}^{2}}{\left(c_{13}^{\prime \prime}\right)^{2}} \sin ^{2} 2 \theta_{13}^{\prime \prime}\left(\frac{c_{\beta}^{2}}{1+\epsilon_{e e}}\right)^{2} \sin ^{2}\left(\frac{\left(1+\epsilon_{e e}\right) A L}{2 c_{\beta}^{2}}\right) .
\end{aligned}
$$

\footnotetext{
${ }^{4}$ Before Ref. [25] appeared, the bounds $\left|\epsilon_{e \mu}^{f P}\right|<\mathcal{O}\left(10^{-4}\right)(90 \%)$ in Ref. [15] based on the one-loop arguments were widely accepted, and this was used to justify the assumption $\epsilon_{e \mu} \simeq 0$ in Refs. [29, 30].
} 
where $c_{13}^{\prime \prime} \equiv \cos \theta_{13}^{\prime \prime}, c_{\beta} \equiv \cos \beta, s_{\beta} \equiv \sin \beta$, and the new angle $\theta_{13}^{\prime \prime}$, which is introduced in the appendix $\mathrm{F}$ to diagonalize the mass matrix in the presence of the new physics, is defined by Eq. (F6).

Comparing Eqs. (6) and (11), we see that if

$$
1+\epsilon_{e e}=c_{\beta}^{2}
$$

is satisfied, then by introducing the two effective mixing angle

$$
\begin{aligned}
\sin ^{2} \theta_{23}^{\mathrm{eff}} & \equiv \frac{s_{23}^{2}}{\left(c_{13}^{\prime \prime}\right)^{2}} \\
\theta_{13}^{\mathrm{eff}} & \equiv \theta_{13}^{\prime \prime},
\end{aligned}
$$

Eq. (11) shows almost the identical behavior as that of the standard scheme (6).

A few remarks are in order.

Firstly, although Eq. (12) is satisfied only in a narrow region 5 as long as Eq. (12) holds, the high-energy behavior of the disappearance oscillation probability coincides with that of the standard three-flavor scheme. Off this upper half circle, equivalence between the behaviors of Eqs. (11) and (6) are lost, but it is expected that due to the experimental errors around this upper half circle, there exist some regions in which the behaviors of Eq. (11) and (6) are similar.

Secondly, Eq. (14) indicates that the angle $\theta_{13}^{\prime \prime}$ plays a role similar to that of $\theta_{13}$ in the standard scheme. Note that the corrections in Eq. (6) due to $\theta_{13}$ were not discussed in Refs. [29, 30], where it was suggested that the quantities that appear in Eq. (11) imply the effective two-flavor mixing angle, $\sin ^{2} \theta_{\text {atm }}=\left(1+t_{\beta}^{2}\right) s_{23}^{2} /\left(1+s_{23}^{2} t_{\beta}^{2}\right)$ and the effective masssquared difference $\Delta m_{\text {atm }}^{2}=\Delta m_{32}^{2}\left(1+s_{23}^{2} t_{\beta}^{2}\right) /\left(1+t_{\beta}^{2}\right)$. While the former is exactly the same as Eq. (13) in the limit $\theta_{13} \rightarrow 0$, the latter does not appear in our result. This is because the correction factor $\left(1+s_{23}^{2} t_{\beta}^{2}\right) /\left(1+t_{\beta}^{2}\right)$ naturally arises from the three-flavor contributions, i.e., from the $\theta_{13}$ dependent terms, and we need not normalize $\Delta m^{2}$. If we postulate the effective mixing angle to be $\theta_{23}^{\text {eff }}=\pi / 4$ in Eq. (13), then $c_{23} \equiv \cos \theta_{23}>0$ can be expressed by $\beta$ and $\theta_{13}$ as

$$
c_{23}=\frac{s_{\beta} c_{\beta} s_{13} c_{13} \cos \Phi}{2-s_{\beta}^{2} c_{13}^{2}}+\left\{\frac{1+c_{\beta}^{2} s_{13}^{2}}{2-s_{\beta}^{2} c_{13}^{2}}+\left(\frac{s_{\beta} c_{\beta} s_{13} c_{13} \cos \Phi}{2-s_{\beta}^{2} c_{13}^{2}}\right)^{2}\right\}^{1 / 2},
$$

where we have introduced

$$
\Phi \equiv \delta+\arg \left(\epsilon_{e \tau}\right)
$$

In the limit $\theta_{13} \rightarrow 0$, Eq. (15) agrees with the expression $c_{23}^{2}=1 /\left(1+c_{\beta}^{2}\right)$ obtained in Refs. [29, 30].

Thirdly, Ref. [60] performed a three-flavor analysis of the atmospheric neutrino data, and the authors concluded that the atmospheric neutrino data alone gives $s_{13}^{2}<0.14(0.27)$ at 90\% CL for a normal (inverted) mass hierarchy. This implies that the range $s_{13}^{2}<0.14$ is consistent at $90 \% \mathrm{CL}$ with the high-energy atmospheric neutrino data, i.e., the upward going $\mu$ events. In the present case, we found that the value of $\left(s_{13}^{\prime \prime}\right)^{2}$ can be made smaller than

\footnotetext{
5 In fact, Eq. (12) stands for an upper half circle around a center $(-1 / 2,0)$ with a radius $1 / 2$ in the $\left(\epsilon_{e e},\left|\epsilon_{e \tau}\right|\right)$
} plane. 
0.14 in almost all the region for $0 \leq \sin ^{2} 2 \theta_{13}<0.15$ and $0 \leq s_{\beta}^{2}<0.5$, by adjusting the value of $\Phi$. With the conditions (12), (13), (14), therefore, the region around the upper half circle for $\left|\epsilon_{e \tau}\right| \lesssim 0.5$ and $-1 / 2 \lesssim 1+\epsilon_{e e} \lesssim 0$ is expected to be consistent with the atmospheric neutrino data.

Thus, taking into account the various constraints described above, we will work with the ansatz

$$
\mathcal{A}=A\left(\begin{array}{ccc}
1+\epsilon_{e e} & 0 & \epsilon_{e \tau} \\
0 & 0 & 0 \\
\epsilon_{e \tau}^{*} & 0 & \left|\epsilon_{e \tau}\right|^{2} /\left(1+\epsilon_{e e}\right)
\end{array}\right)
$$

in the following discussions.

\section{SENSITIVITY OF T2KK TO $\epsilon_{e e}$ AND $\epsilon_{e \tau}$}

In this section we discuss the sensitivity of the T2KK experiment to the non-standard interaction in propagation with the ansatz (16). Since $\epsilon_{\tau \tau}$ is expressed in terms of $\epsilon_{e \tau}$ and $\epsilon_{e e}$, the only new degrees of freedom are $\epsilon_{e e},\left|\epsilon_{e \tau}\right|$ and $\arg \left(\epsilon_{e \tau}\right)$. Firstly, in sect.IIA, we briefly describe the setup of the T2KK experiment. Secondly, in sect.IIIB, we consider the $\left(\epsilon_{e e},\left|\epsilon_{e \tau}\right|\right)$ plane and discuss the region in which T2KK can discriminate the non-standard interaction in propagation from the standard three-flavor scenario. Thirdly, in sect.IIIC, we study the case in which new physics can be discriminated and discuss how precisely T2KK can determine $\epsilon_{e e}$ and $\left|\epsilon_{e \tau}\right|$. Then, in sect.IIID, we consider whether the two complex phases $\delta$ and $\arg \left(\epsilon_{e \tau}\right)$ can be determined separately.

\section{A. The T2KK experiment}

The T2KK experiment [42, 43] is a proposal for the future extension of the T2K experiment [44]. In this proposal, a water Cherenkov detector is placed not only in Kamioka (at a baseline length $L=295 \mathrm{~km}$ ) but also in Korea (at $L=1050 \mathrm{~km}$ ), whereas the power of the beam at J-PARC in Tokai Village is upgraded to $4 \mathrm{MW}$. As in the T2K experiment, it is assumed that T2KK uses an off-axis beam with a $2.5^{\circ}$ angle between the directions of the charged pions and neutrinos, and the neutrino energy spectrum has a peak approximately at $0.7 \mathrm{GeV}$. Because the two detectors are assumed to be identical, some of the systematic errors cancel. Also, because the distances of the two detectors from the source are different, parameter degeneracy in the three-flavor oscillation scenario [55 58] is expected to be resolved with this complex [42, 43, 61 64].

In this paper, we assume the same setup as that in Refs. [41, 62]. In our analysis, we use the disappearance channel $\nu_{\mu} \rightarrow \nu_{\mu}$ and $\bar{\nu}_{\mu} \rightarrow \bar{\nu}_{\mu}$, the appearance one $\nu_{\mu} \rightarrow \nu_{e}$ and $\bar{\nu}_{\mu} \rightarrow \bar{\nu}_{e}$, and data from single-Cherenkov-ring electron and muon events. We assume that the measurement will run for 8 years in total, 4 years each for the neutrino and antineutrino beams. The fiducial volume of each detector is 0.27 Mton. The density of the Earth is assumed to be $\rho=2.3\left[\mathrm{~g} / \mathrm{cm}^{3}\right]$ in the case of Tokai-Kamioka, and $\rho=2.8\left[\mathrm{~g} / \mathrm{cm}^{3}\right]$ in the case of Tokai-Korea. The electron fraction $Y_{e}$ is assumed to be 0.5. The energy resolution is considered to be $80 \mathrm{MeV}$. We use various information such as the neutrino flux from Ref. 65]. 


\section{B. Bounds on $\epsilon_{e e}$ and $\epsilon_{e \tau}$}

Firstly, we discuss the case of the region $\left(\epsilon_{e e},\left|\epsilon_{e \tau}\right|\right)$, in which we can distinguish the new physics with ansatz (16) from the standard three-flavor scheme. To perform such a test, we introduce the following quantity:

$$
\begin{aligned}
\Delta \chi^{2}=\min _{\text {param }, \epsilon_{l}}\left[\sum_{k=1}^{4}\{\right. & \sum_{i=1}^{5} \frac{1}{\sigma_{i}^{2}(e)}\left\{N_{i}^{0}(e)+B_{i}^{0}(e)-N_{i}(e) \sum_{l=3,7}\left(1+f(e)_{l}^{i} \epsilon_{l}\right)\right. \\
& \left.-B_{i}(e) \sum_{l=1,2,7}\left(1+f(e)_{l}^{i} \epsilon_{l}\right)\right\}^{2} \\
& +\sum_{i=1}^{20} \frac{1}{\sigma_{i}^{2}(\mu)}\left\{N_{i}^{0}(\mu)+B_{i}^{0}(\mu)-N_{i}(\mu) \sum_{l=4,5,7}\left(1+f(\mu)_{l}^{i} \epsilon_{l}\right)\right. \\
& \left.\left.-B_{i}(\mu) \sum_{l=4,6,7}\left(1+f(\mu)_{l}^{i} \epsilon_{l}\right)\right\}^{2}\right\} \\
+ & \left.\sum_{l=1}^{7}\left(\frac{\epsilon_{l}}{\tilde{\sigma}_{l}}\right)^{2}+\Delta \chi_{\text {prior }}^{2}\right]
\end{aligned}
$$

where the prior $\Delta \chi_{\text {prior }}^{2}$ is given by

$$
\Delta \chi_{\text {prior }}^{2} \equiv \frac{2.7 \times\left(\sin ^{2} 2 \theta_{23}-1.0\right)^{2}}{(0.06)^{2}}+\frac{\left(\sin ^{2} \theta_{13}-0.02\right)^{2}}{(0.01)^{2}}+\frac{\left(\left|\Delta m_{31}^{2}\right|-2.4 \times 10^{-3}\left[\mathrm{eV}^{2}\right]\right)^{2}}{\left(1.5 \times 10^{-4}\left[\mathrm{eV}^{2}\right]\right)^{2}}
$$

In principle we could perform an analysis without the prior $\Delta \chi_{\text {prior }}^{2}$, but in that case it would take more computation time by minimizing $\Delta \chi^{2}$ for the parameter region which is already excluded by the present data of the atmospheric and reactor experiments. So we have included the prior in our analysis to save computation time. In Eq. (17), $N_{i}^{0}(e)$, $N_{i}^{0}(\mu)\left(B_{i}^{0}(e)\right.$, and $\left.B_{i}^{0}(\mu)\right)$ are the expected signal (background) numbers of events in the presence of the new physics (16), while $N_{i}(e), N_{i}(\mu)\left(B_{i}(e)\right.$, and $\left.B_{i}(\mu)\right)$ are the expected signal (background) numbers of events in the three-flavor framework with the standard matter effect. All these numbers except $B_{i}^{0}(e)$ and $B_{i}(e)$ depend on the neutrino oscillation parameters. The indices $i$ and $k=1, \cdots, 4$ stand for the number of the neutrino energy bin for electrons and muons and the four combinations of detectors in Kamioka and Korea with the neutrino and anti-neutrino beams, respectively. For the electron events, there are five energy bins (400-500 MeV, 500-600 MeV, 600-700 MeV, 700-800 MeV, and 800-1200 MeV), whereas for the muon events, there are twenty bins from 200 to $1200 \mathrm{MeV}$ with $50 \mathrm{MeV}$ width. $\sigma_{i}(\ell)(\ell=e, \mu)$ stands for the statistical uncertainties, whereas $\epsilon_{\ell}(\ell=1, \cdots, 7)$ stands for the systematic uncertainties in the expected number of signals and backgrounds. $\Delta \chi^{2}$ is defined by minimizing the quantity inside the square bracket in Eq. (17) with respect to the uncertainties $\epsilon_{\ell}$ as well as the oscillation parameters $\left(\left|\Delta m_{31}^{2}\right|, \operatorname{sign}\left(\Delta m_{31}^{2}\right), \theta_{23}, \delta\right)$ of the standard three-flavor scheme, on which the numbers of events $N_{i}(e), N_{i}(\mu), B_{i}(e)$, and $B_{i}(\mu)$ depend. The uncertainties in $B_{i}(e)$ and $N_{i}(e)$ are represented by 4 parameters $\epsilon_{j}(j=$ $1,2,3,7)$. The backgrounds in the muon events are referred to as non-quasi-elastic events in Refs. [41, 62]. The uncertainties in $B_{i}(\mu)$ and $N_{i}(\mu)$ are represented by 4 parameters $\epsilon_{j}(j=$ $4, \cdots, 7)$. The parameter $f(e \text { or } \mu)_{j}^{i}$ indicates the possible dependence of the parameter $\epsilon_{j}$ on the $i$-th energy bin. $\epsilon_{1}$ stands for the uncertainty in the overall background normalization for 
electron events with $\tilde{\sigma}_{1}=0.05 . \epsilon_{2}$ is the energy-dependent uncertainty for the background electron-like events with a function $f(e)_{2}^{i}=\left(\left(E_{\nu}-800 \mathrm{MeV}\right) / 400 \mathrm{MeV}\right)$ and $\tilde{\sigma}_{2}=0.05 . \epsilon_{3}$ is the uncertainty in the detection efficiency for the electron signal events with $\tilde{\sigma}_{3}=0.05$. $\epsilon_{4}$ is the energy-dependent uncertainty for both the muon signal and background events with the function $f(\mu)_{4}^{i}=\left(E_{\nu}-800 \mathrm{MeV}\right) / 800 \mathrm{MeV}$ and $\tilde{\sigma}_{4}=0.05 . \epsilon_{5}$ is the uncertainty in the signal detection efficiency for the muon signal events with $\tilde{\sigma}_{5}=0.05$. $\epsilon_{6}$ is the uncertainty in the separation of quasi-elastic and non-quasi-elastic interactions in the muon events and $\tilde{\sigma}_{6}=0.20 . \epsilon_{7}$ stands for the uncertainty in the neutrino flux in Korea, and $\tilde{\sigma}_{7}$ is assumed to be the predicted flux difference between those in Kamioka and in Korea, given in Ref. [66].

In Eq. (17), the numbers of events $N_{i}^{0}(e), N_{i}^{0}(\mu)\left(B_{i}^{0}(e)\right.$, and $\left.B_{i}^{0}(\mu)\right)$ depend not only on the new physics parameters $\epsilon_{e e},\left|\epsilon_{e \tau}\right|, \arg \left(\epsilon_{e \tau}\right)$ but also on the standard oscillation parameters, which we denote as $\bar{\theta}_{12}, \bar{\theta}_{13}, \bar{\theta}_{23}, \Delta \bar{m}_{21}^{2}, \Delta \bar{m}_{31}^{2}$, and $\bar{\delta}$. Here, we take the best-fit values for most of the standard oscillation parameters as the reference values:

$$
\begin{aligned}
\sin ^{2}\left(2 \bar{\theta}_{12}\right) & =0.87 \\
\sin ^{2}\left(2 \bar{\theta}_{23}\right) & =1.0 \\
\Delta \bar{m}_{21}^{2} & =7.9 \times 10^{-5} \mathrm{eV}^{2} \\
\Delta \bar{m}_{32}^{2} & =2.4 \times 10^{-3} \mathrm{eV}^{2}
\end{aligned}
$$

On the other hand, since we have no information on $\bar{\theta}_{13}$ and $\bar{\delta}$, we will take several reference values for these parameters.

The results are shown in Figs.14, where the curves are drawn at 90\% CL $\left(\Delta \chi^{2}=4.6\right.$ for 2 degrees of freedom). The new physics with the ansatz (16) can be distinguished from the standard three-flavor scheme outside the curves. Four different choices for the phases $\delta$ and $\arg \left(\epsilon_{e \tau}\right)$ are taken, where the sum of the two phases is the same in each figure. It has been known [48, 67] that in the limit of $\Delta m_{21}^{2} \rightarrow 0$, the oscillation probability $P\left(\nu_{\mu} \rightarrow \nu_{e}\right)$ depends only on the combination $\delta+\arg \left(\epsilon_{e \tau}\right)$ of the phases. If the four curves in Figs,14 coincided with each other, then it would mean that the contribution of the solar masssquared difference were small. From these figures, however, the behaviors of the four curves are different even if $\delta+\arg \left(\epsilon_{e \tau}\right)=$ constant, so the contribution of $\Delta m_{21}^{2}$ is not negligible. This is because we are considering the oscillation probabilities in Korea, where $\Delta m_{21}^{2} L / 4 E \sim 0.3$. The analytic form of the oscillation probability $P\left(\nu_{\mu} \rightarrow \nu_{e}\right)$ is given in the appendix $\mathbb{E}$, where the correction to $P\left(\nu_{\mu} \rightarrow \nu_{e}\right)$ to the first order in $\Delta m_{21}^{2}$ is also given. The corrections to the energy eigenvalues $\tilde{E}_{j}(j=1,2,3)$ are proportional to either $\sin ^{2} \theta_{12}^{\prime \prime}$ or $\cos ^{2} \theta_{12}^{\prime \prime}$, where $\theta_{12}^{\prime \prime}$ is defined in Eq. (F7), and $\theta_{12}^{\prime \prime}$ depends mainly on $\arg \left(\epsilon_{e \tau}\right)$. From these figures we conclude that the T2KK experiment can distinguish the new physics with ansatz (16) at 90\% CL approximately for $\left|\epsilon_{e e}\right| \gtrsim 1$ and $\left|\epsilon_{e \tau}\right| \gtrsim 0.2$. In other words, if T2KK lacks evidence of a new physics, then with the ansatz (16), we can put bounds on the two parameters: $\left|\epsilon_{e e}\right| \lesssim 1$ and $\left|\epsilon_{e \tau}\right| \lesssim 0.2$. While the bound on $\epsilon_{e e}$ is modest, the one on $\left|\epsilon_{e \tau}\right|$ is impressive compared with the present bound (3).

\section{Precision in determination of $\epsilon_{e e},\left|\epsilon_{e \tau}\right|$}

Let us now turn to the case with affirmative results in the T2KK experiment, i.e., we will discuss the points that lie outside the curves in the $\left(\epsilon_{e e},\left|\epsilon_{e \tau}\right|\right)$ plane in Figs 1 4.6

6 Since we have the fixed value of $\theta_{13}$, we do not have the $\theta_{13}-\epsilon_{e \tau}$ confusion in Ref. [49], in which it was shown that the same neutrino survival probability is produced by the different pairs of $\left(\theta_{13}, \epsilon_{e \tau}\right)$. 

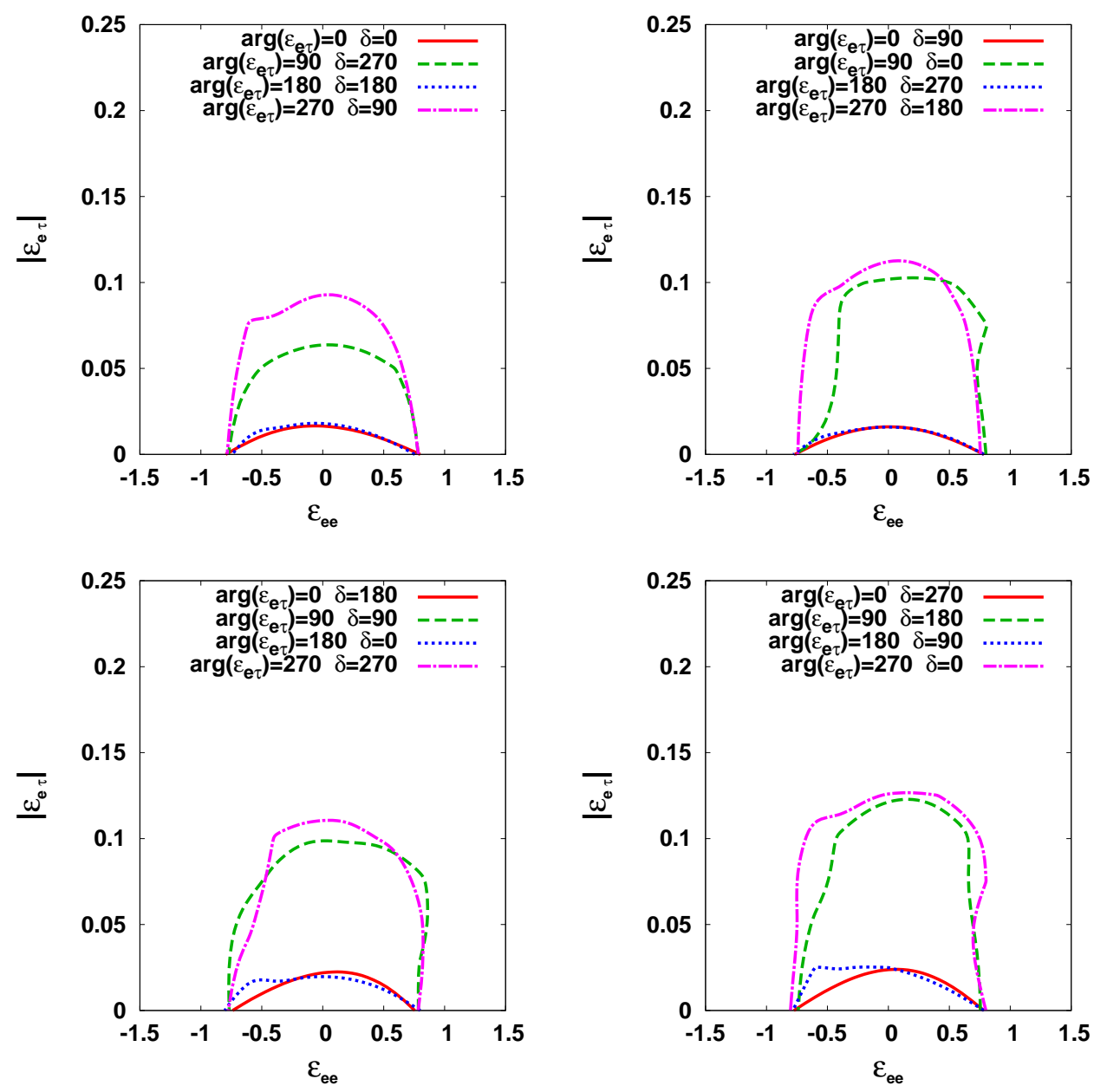

FIG. 1. Region in which the new physics is discriminated at 90\%CL from the standard three-flavor scheme for $\sin ^{2} 2 \bar{\theta}_{13}=10^{-4}$.

First, we discuss the experimental errors in $\epsilon_{e e}$ and $\left|\epsilon_{e \tau}\right|$, i.e., the correlation of errors for these two variables. In this case, we introduce the following quantity, which is similar to Eq. (17):

$$
\begin{aligned}
\Delta \chi^{2}=\min _{\text {param }, \epsilon_{\ell}}\left[\sum_{k=1}^{4}\{\right. & \sum_{i=1}^{5} \frac{1}{\sigma_{i}^{2}(e)}\left\{N_{i}^{0}(e)+B_{i}^{0}(e)-N_{i}(e) \sum_{l=3,7}\left(1+f(e)_{l}^{i} \epsilon_{l}\right)\right. \\
& \left.-B_{i}(e) \sum_{l=1,2,7}\left(1+f(e)_{l}^{i} \epsilon_{l}\right)\right\}^{2} \\
& +\sum_{i=1}^{20} \frac{1}{\sigma_{i}^{2}(\mu)}\left\{N_{i}^{0}(\mu)+B_{i}^{0}(\mu)-N_{i}(\mu) \sum_{l=4,5,7}\left(1+f(\mu)_{l}^{i} \epsilon_{l}\right)\right. \\
& \left.\left.-B_{i}(\mu) \sum_{l=4,6,7}\left(1+f(\mu)_{l}^{i} \epsilon_{l}\right)\right\}^{2}\right\} \\
+ & \left.\sum_{l=1}^{7}\left(\frac{\epsilon_{l}}{\tilde{\sigma}_{l}}\right)^{2}\right]
\end{aligned}
$$

where most of the definitions are the same as those in Eq. (17). The only differences between 

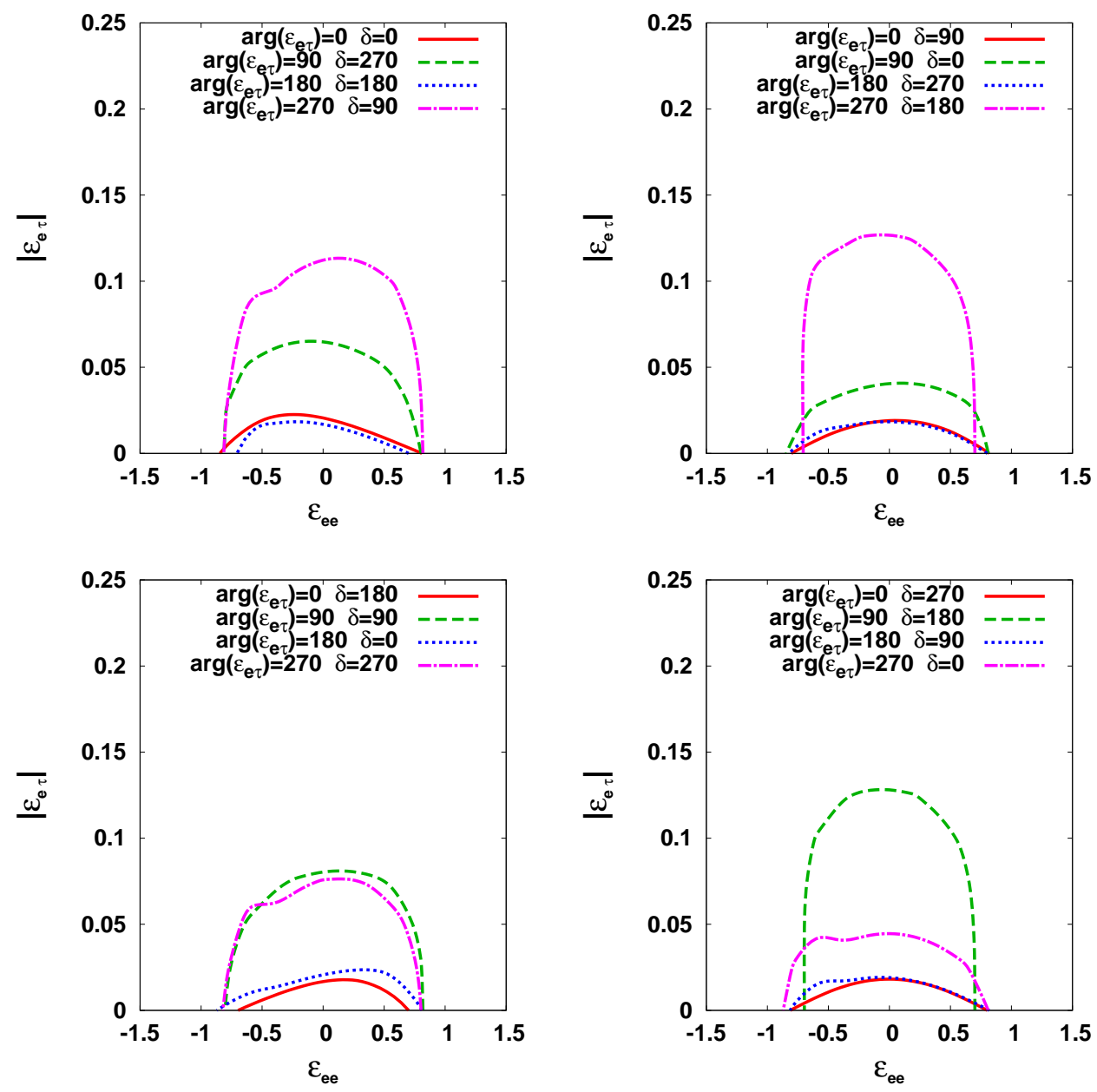

FIG. 2. Region in which the new physics is discriminated at 90\% CL from the standard three-flavor scheme for $\sin ^{2} 2 \bar{\theta}_{13}=10^{-3}$.

(17) and (19) are that the prior is absent in the latter, and that $N_{i}(\ell)(\ell=e, \mu)$ and $B_{i}(\ell)(\ell=e, \mu)$ in the latter (in the former) are the expected numbers of events in the presence of new physics with ansatz (16) (in the standard scheme), respectively. Namely, both $N_{i}^{0}(\ell)(\ell=e, \mu)$ and $\left(B_{i}^{0}(\ell)(\ell=e, \mu)\right.$ and are the expected number of events in the presence of a new physics with the ansatz (16) in Eq. (19). The number of events $N_{i}^{0}(\ell)(\ell=e, \mu)$ depends on the parameters of the new physics $\left(\bar{\epsilon}_{e e},\left|\bar{\epsilon}_{e \tau}\right|\right.$, and $\left.\arg \left(\bar{\epsilon}_{e \tau}\right)\right)$ as well as the oscillation parameters of the standard scheme $\bar{\theta}_{12}, \bar{\theta}_{13}, \bar{\theta}_{23}, \Delta \bar{m}_{21}^{2}, \Delta \bar{m}_{31}^{2}$, and $\bar{\delta}$. We fix $\bar{\epsilon}_{e e},\left|\bar{\epsilon}_{e \tau}\right|$ at some points outside the curves in Figs,14 and evaluate $\Delta \chi^{2}$ as a function of $\epsilon_{e e}$ and $\left|\epsilon_{e \tau}\right|$, which appear in the argument of $N_{i}(\ell)(\ell=e, \mu)$. For simplicity, we assume the central values given in Eqs. (18) for $\bar{\theta}_{12}, \bar{\theta}_{23}, \Delta \bar{m}_{21}^{2}$, and $\Delta \bar{m}_{31}^{2}$. For $\bar{\theta}_{13}$, we take a few representative values $\sin ^{2} 2 \bar{\theta}_{13}=10^{-4}, 10^{-2}, 0.12$. We assume normal hierarchy and fix the value of the phases as $\bar{\delta}=\pi$ and $\arg \left(\bar{\epsilon}_{e \tau}\right)=\pi$ for simplicity. As for the variables in $N_{i}(\ell)(\ell=e, \mu)$, for simplicity we equate the variables $\theta_{12}, \theta_{13}, \theta_{23}, \Delta m_{21}^{2}, \Delta m_{31}^{2}, \delta$, and $\arg \left(\epsilon_{e \tau}\right)$ to $\bar{\theta}_{12}, \bar{\theta}_{13}, \bar{\theta}_{23}, \Delta \bar{m}_{21}^{2}, \Delta \bar{m}_{31}^{2}, \bar{\delta}$, and $\arg \left(\bar{\epsilon}_{e \tau}\right)$ in $N_{i}^{0}(\ell)(\ell=e, \mu)$, respectively. In this analysis, we do not introduce any prior because it will be difficult to estimate it in the presence of the new physics. Thus, in Eq. (19), we only minimize the quantity in the square 

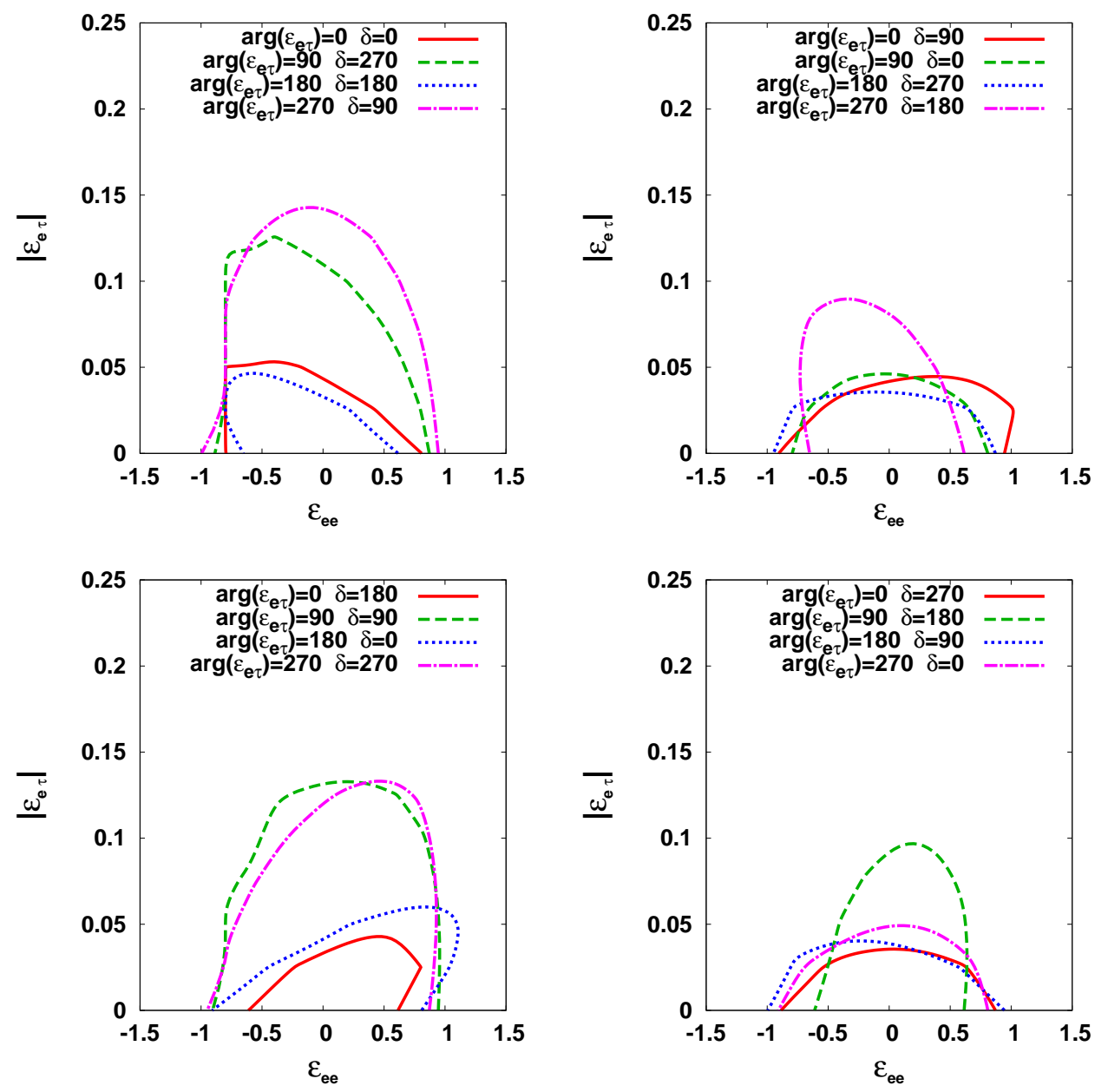

FIG. 3. Region in which the new physics is discriminated at 90\%CL from the standard three-flavor scheme for $\sin ^{2} 2 \bar{\theta}_{13}=10^{-2}$.

bracket with respect to the parameters $\epsilon_{\ell}(\ell=1, \cdots, 7)$, and we evaluate $\Delta \chi^{2}$ as a function of the variables $\epsilon_{e e}$ and $\left|\epsilon_{e \tau}\right|$.

The results are shown in Fig 5 , where the contours around the true points are depicted at $90 \% \mathrm{CL}\left(\Delta \chi^{2}=4.6\right.$ for 2 degrees of freedom) for three different values of $\theta_{13}$. The straight lines $\left|\epsilon_{e \tau}\right|=1.1 \times\left|1+\epsilon_{e e}\right|$ in Fig 5 stand for the approximate bound from the atmospheric neutrinos, which lead to $\cos ^{2} \beta>0.45$ or $|\tan \beta| \lesssim 1.1[30]$, and we have examined only the points below these straight lines. As seen from Fig.5, the error in $\left|\epsilon_{e \tau}\right|$ is relatively smaller compared with that in $\epsilon_{e e}$ for all the values of $\theta_{13}$. The experimental error in $\epsilon_{e e}$ increases for smaller values of $\left|\epsilon_{e \tau}\right|$ and $\theta_{13}$. For $\left|\epsilon_{e \tau}\right| \lesssim 0.3$, therefore, the possibility of $\epsilon_{e e}=0$ cannot be excluded by the experiment, while $\left|\epsilon_{e \tau}\right|=0$ can be for $\left|\epsilon_{e \tau}\right| \gtrsim 0.2$. For $1+\epsilon_{e e}<0$, the correlation in $\epsilon_{e e}$ and $\left|\epsilon_{e \tau}\right|$ increases, and in this case, even if T2KK can discriminate the new physics from the standard scheme, the determination of these parameters is difficult. We have also analyzed other combinations of the phases $\delta$ and $\arg \left(\epsilon_{e \tau}\right)$ and they share the same features as in Fig. 5 . 

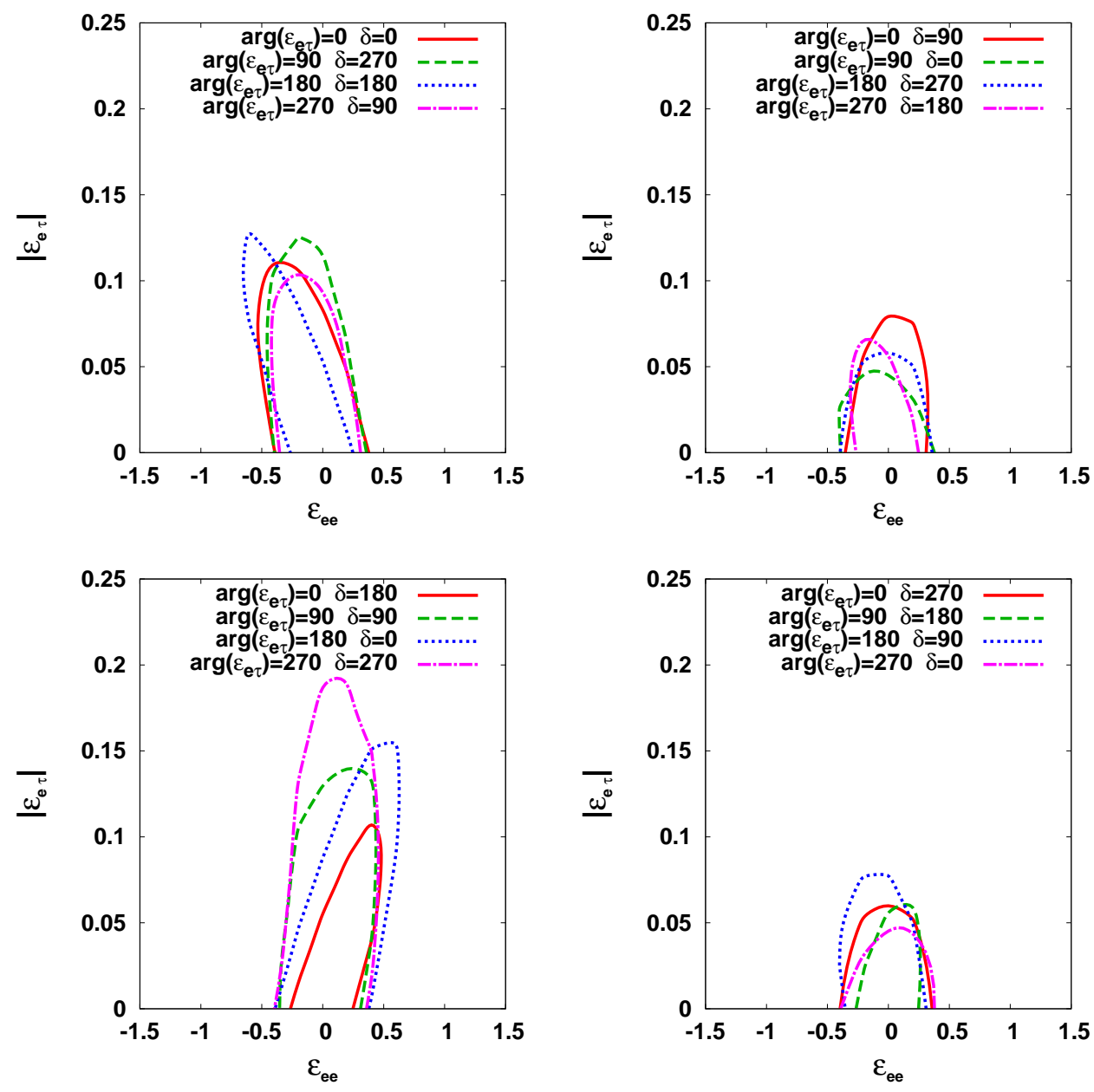

FIG. 4. Region in which the new physics is discriminated at 90\% CL from the standard three-flavor scheme for $\sin ^{2} 2 \bar{\theta}_{13}=0.12$.

\section{CP violating phases}

In the ansatz (16), there are two phases $\delta$ and $\arg \left(\bar{\epsilon}_{e \tau}\right)$, and if a new physics exists at all, then it becomes important whether we can determine these two phases separately. Thus, we address this question next. In this case, we can use the same $\Delta \chi^{2}$ in Eq. (19), but there are two differences between this and sectஹIC. Firstly, here we vary the variables $\epsilon_{e e}$ and $\left|\epsilon_{e \tau}\right|$ in $N_{i}(\ell)(\ell=e, \mu)$ and minimize the quantity in the square bracket in Eq. (19) with respect to these two parameters as well as the parameters $\epsilon_{\ell}$. Secondly, $\Delta \chi^{2}$ is plotted as a function of the two variables $\delta$ and $\arg \left(\bar{\epsilon}_{e \tau}\right)$ in $N_{i}(\ell)(\ell=e, \mu)$ here, while it is plotted as a function of the two variables $\epsilon_{e e}$ and $\left|\epsilon_{e \tau}\right|$ in $N_{i}(\ell)(\ell=e, \mu)$ in sect IIIC.

The results at 90\% CL are shown in Figs. 6] and 7 for $\left(\bar{\epsilon}_{e e},\left|\bar{\epsilon}_{e \tau}\right|\right)=(0.8,0.2)$ and $(2.0,2.0)$, respectively. Since we are discussing the cases that can be distinguished from the standard scheme, relatively large values of $\left|\bar{\epsilon}_{e \tau}\right|$ are chosen in both examples. Again $\bar{\delta}=\arg \left(\bar{\epsilon}_{e \tau}\right)=\pi$ is assumed for the true values of the phases. To clarify the roles of the detectors at the two baseline lengths, separate contours are given for the result from the detector in Kamioka, for that from the detector in Korea, and for that from the combination of the two. As 


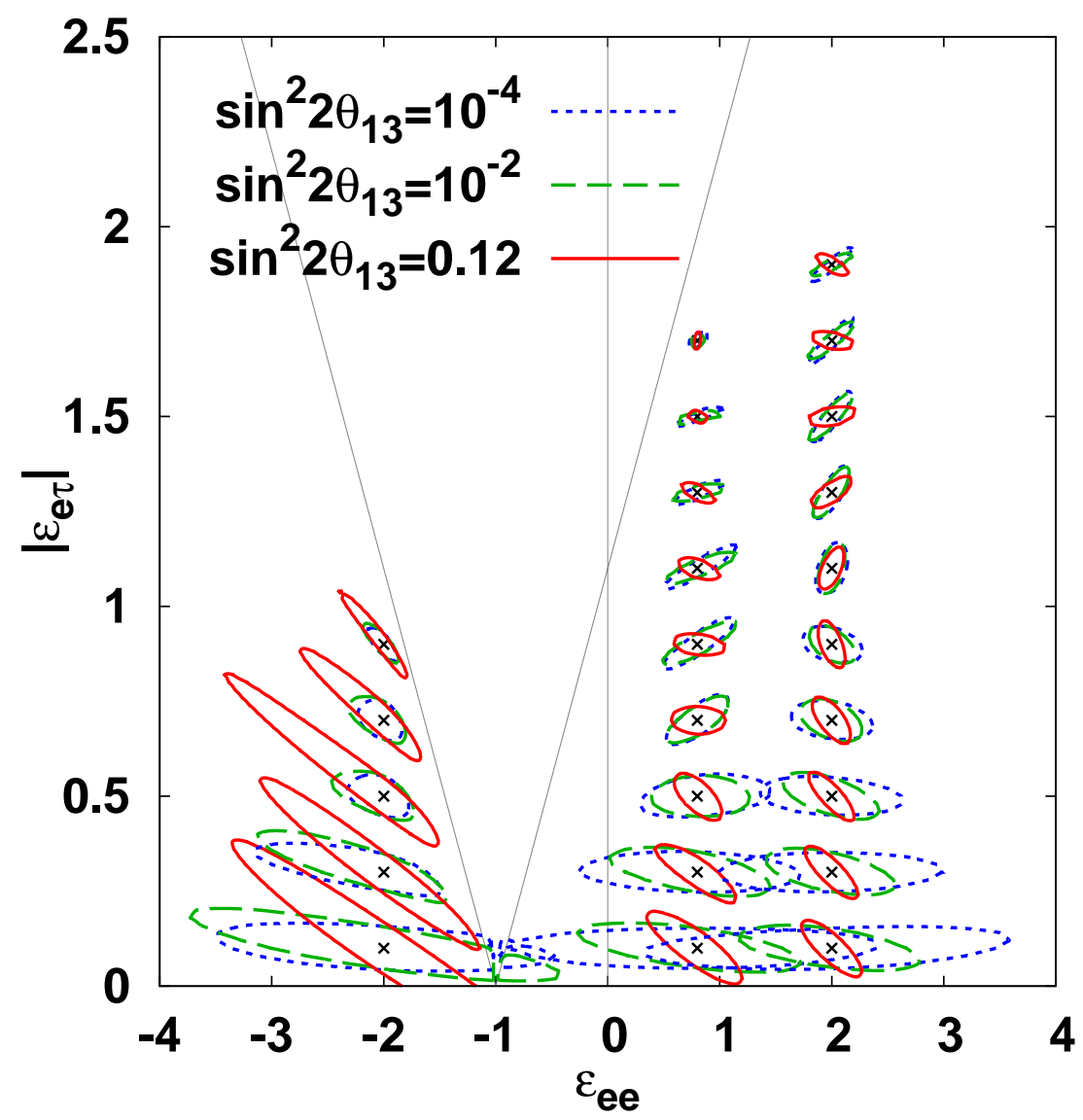

FIG. 5. Correlation in $\left(\epsilon_{e e},\left|\epsilon_{e \tau}\right|\right)$ : The contours around the true points are depicted at 90\%CL. $\bar{\delta}=\arg \left(\bar{\epsilon}_{e \tau}\right)=\pi$ is assumed.

in the standard three-flavor case, if $\theta_{13}$ is very small, neither the detector in Kamioka nor the one in Korea can provide any information on $\delta$. As the value of $\theta_{13}$ increases, the sensitivity to $\delta$ of the detectors in Kamioka and Korea increases. For larger values of $\theta_{13}$, the sensitivity to $\arg \left(\epsilon_{e \tau}\right)$ depends on the value of $\left|\epsilon_{e \tau}\right|$. For larger (smaller) values of $\left|\epsilon_{e \tau}\right|$, sensitivity to $\arg \left(\epsilon_{e \tau}\right)$ is good (poor). These features can be understood qualitatively by looking at the $\mathrm{T}$ violating term in the oscillation probability $P\left(\nu_{\mu} \rightarrow \nu_{e}\right)$ (see appendix $\mathrm{G}$ for details). In the present case, there are two sources for $\mathrm{T}$ violation, the standard one $\operatorname{Im}\left\{Y_{3}^{\mu e}\left(Y_{2}^{\mu e}\right)^{*}\right\}_{\text {std }}$ and the extra one $\operatorname{Im}\left\{Y_{3}^{\mu e}\left(Y_{2}^{\mu e}\right)^{*}\right\}_{\text {NP }}$ because of the new physics. From the explicit forms (G3) and (G4), the ratio of the two terms is roughly given by (std)/(NP) $\left|\Delta E_{31}\right|\left|U_{e 3}\right| /\left(A\left|\epsilon_{e \tau}\right|\left|U_{\tau 3}\right|\right) \sim 10 s_{13} /\left|\epsilon_{e \tau}\right|$. This implies that if $\sin ^{2} 2 \theta_{13} \gtrsim(\lesssim) \mathcal{O}\left(10^{-3}\right)$ and if $\left|\epsilon_{e \tau}\right|=0.2$, then the contribution of $\theta_{13}$ is large (small). If $\left|\epsilon_{e \tau}\right|$ is very large, then the oscillation probability and the number of events becomes so large that the sensitivity of both the detectors to the two phases increases, as shown in Fig. 7. 


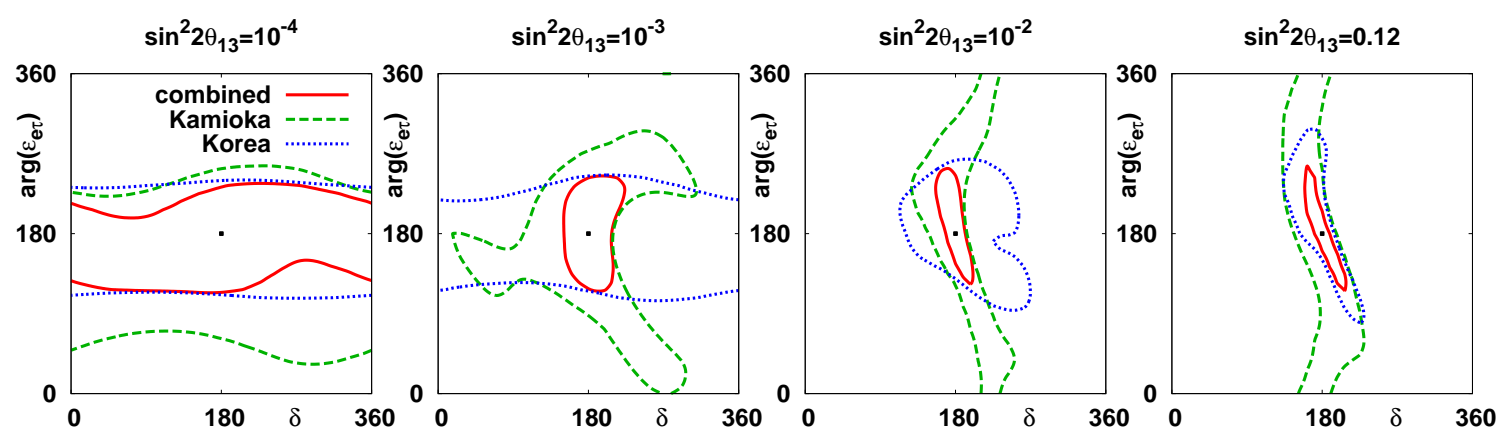

FIG. 6. Correlation in $\left(\delta, \arg \left(\epsilon_{e \tau}\right)\right)$ for $\epsilon_{e e}=0.8,\left|\epsilon_{e \tau}\right|=0.2$. The contours around the true points are depicted at $90 \% \mathrm{CL}$.

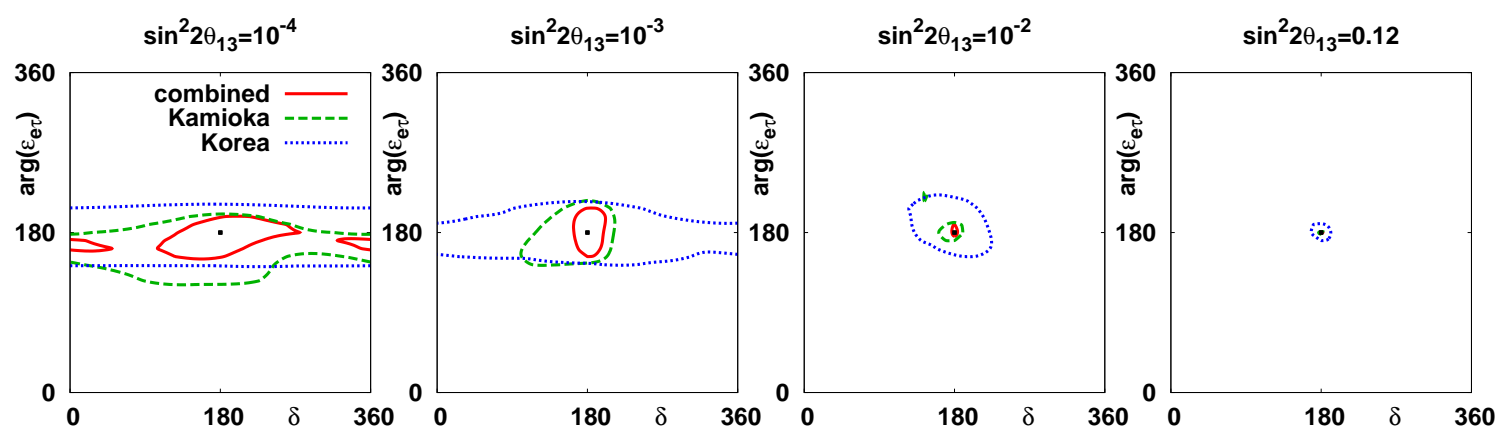

FIG. 7. Correlation in $\left(\delta, \arg \left(\epsilon_{e \tau}\right)\right)$ for $\epsilon_{e e}=2.0,\left|\epsilon_{e \tau}\right|=2.0$. The contours around the true points are depicted at $90 \% \mathrm{CL}$.

\section{CONCLUSIONS}

In this paper we have studied the sensitivity of the T2KK experiment to the non-standard interaction in propagation with the ansatz (16).

To justify our premise, we have provided an argument that $\epsilon_{\mu \alpha}(\alpha=e, \mu, \tau)$ must be small for the behavior of the disappearance probability to be consistent with the high-energy atmospheric neutrino data. Using the analytical form of the disappearance probability, we showed that $\epsilon_{\mu \alpha}(\alpha=e, \mu, \tau) \simeq 0$ as well as $\epsilon_{\tau \tau} \simeq\left|\epsilon_{e \tau}\right|^{2} /\left(1+\epsilon_{e e}\right)$ are necessary for the disappearance probability to be consistent with the high-energy behavior $1-P\left(\nu_{\mu} \rightarrow \nu_{\mu}\right) \propto$ $1 / E^{2}$ in the high-energy atmospheric neutrino data. This speculation should be verified explicitly by numerical calculations in the future.

With the ansatz (16), we have looked for the region in the $\left(\epsilon_{e e},\left|\epsilon_{e \tau}\right|\right)$ plane in which T2KK can distinguish the new physics from the standard three-flavor scheme. At 90\%CL T2KK can discriminate the new physics from the standard case for approximately $\left|\epsilon_{e e}\right| \gtrsim 1$ and $\left|\epsilon_{e \tau}\right| \gtrsim 0.2$. These values can be interpreted as bounds for these parameters, if T2KK has negative evidence for a new physics. While the bound on $\epsilon_{e e}$ by T2KK is modest, the bound on $\left|\epsilon_{e \tau}\right|$ by T2KK is much stronger than the present one $\left|\epsilon_{e \tau}\right| \lesssim 3$, and the latter is also stronger than those by other on-going long-baseline experiments such as MINOS or OPERA: If $\sin ^{2} 2 \theta_{13} \simeq 0.07$, then MINOS will give a bound $\left|\epsilon_{e \tau}\right| \lesssim 1$ at 90\%CL 35-37], and the combination of MINOS, OPERA and Double-CHOOZ gives a bound $-2.2(-2.5) \lesssim$ 
$\epsilon_{e \tau} \lesssim 0.6(0.5)$ at $95 \%$ CL for $\sin ^{2} 2 \theta_{13}=0.05(0.1)$ [39].

On the other hand, if the true values of the new physics parameters lie much outside these bounds, then T2KK can determine the values of $\epsilon_{e e},\left|\epsilon_{e \tau}\right|$, and $\arg \left(\epsilon_{e \tau}\right)$. In particular, if the values of $\theta_{13}$ and $\left|\epsilon_{e \tau}\right|$ are relatively large $\left(\sin ^{2} 2 \theta_{13} \gtrsim \mathcal{O}(0.01),\left|\epsilon_{e \tau}\right| \gtrsim 0.2\right)$, then we can determine the two phases $\delta, \arg \left(\epsilon_{e \tau}\right)$ separately. This determination is possible, because the oscillation probability in Korea with the baseline $L=1050 \mathrm{~km}$ receives a nonnegligible contribution from the solar mass-squared difference $\Delta m_{21}^{2}$, and it induces terms that approximately depend only on $\arg \left(\epsilon_{e \tau}\right)$.

Since our analysis is based on the ansatz (16), all the results derived in this paper are approximate ones. Nethertheless we believe that our conclusions are valid.

Long-baseline neutrino experiments with longer baselines $(L \gtrsim 1000 \mathrm{~km})$ are sensitive to the matter effect because of the matter effect contribution appears in the form of $A L / 2 \sim$ $L / 4000 \mathrm{~km}$ in the argument of a sine function in the oscillation probability. They are, therefore, also sensitive to a non-standard interaction in propagation. Among long-baseline experiments with longer baselines, T2KK, for which the matter effect and the contribution from the solar mass-squared difference are smaller than the one from the atmospheric masssquared difference $\left(\left|\Delta E_{21}\right| \sim A \ll\left|\Delta E_{31}\right|\right)$, is different from neutrino factories, for which the contribution from the solar mass-squared difference is smaller than the matter effect and the one from the atmospheric mass-squared difference $\left(\left|\Delta E_{21}\right| \ll A \sim\left|\Delta E_{31}\right|\right)$. Consequently, the features of $\mathrm{T} 2 \mathrm{KK}$ can complement those of neutrino factories. While T2KK is known to be powerful in resolving parameter degeneracy in the standard three-flavor scenario, further studies on the new physics potential of T2KK should be pursued.

\section{Appendix A: Analytic treatment of the oscillation probability in constant density matter}

Throughout this paper, we assume that the density of matter is constant. In this appendix we derive analytically the neutrino oscillation probability in constant density matter. Let us start with the Dirac equation

$$
i \frac{d \Psi}{d t}=\left(U \mathcal{E} U^{-1}+\mathcal{A}\right) \Psi
$$

where $\Psi \equiv\left(\nu_{e}, \nu_{\mu}, \nu_{\tau}\right)^{T}$ is the flavor eigenstate,

$$
\mathcal{E} \equiv \operatorname{diag}\left(E_{1}, E_{2}, E_{3}\right),
$$

is a diagonal matrix with the energy eigenvalues in vacuum, and we assume the nonstandard matter potential $\mathcal{A}$ defined in Eq. (2). In practical calculations, it is easier to subtract the mass matrix $U \mathcal{E} U^{-1}+\mathcal{A}$ by $E_{1} \mathbf{1}$, which only affects the phase of the oscillation amplitude. In the following, therefore, by Eq. (A2) we mean

$$
\mathcal{E} \equiv \operatorname{diag}\left(E_{1}, E_{2}, E_{3}\right)-E_{1} \mathbf{1}=\operatorname{diag}\left(0, \Delta E_{21}, \Delta E_{31}\right),
$$

where $\Delta E_{j k} \equiv E_{j}-E_{k} \simeq \Delta m_{j k}^{2} / 2 E$.

The $3 \times 3$ matrix on the right hand side of the Dirac equation (A1) can be formally diagonalized as:

$$
U \mathcal{E} U^{-1}+\mathcal{A}=\tilde{U} \tilde{\mathcal{E}} \tilde{U}^{-1}
$$


where

$$
\tilde{\mathcal{E}} \equiv \operatorname{diag}\left(\tilde{E}_{1}, \tilde{E}_{2}, \tilde{E}_{3}\right)
$$

is a diagonal matrix with the energy eigenvalues in matter.

As in the case of the oscillation probability in vacuum, Eq. (A1) can be integrated as

$$
\Psi(L)=\tilde{U} \exp (-i \tilde{\mathcal{E}} L) \tilde{U}^{-1} \Psi(0)
$$

Thus the oscillation probability $P\left(\nu_{\alpha} \rightarrow \nu_{\beta}\right)$ is given by

$$
\begin{aligned}
P\left(\nu_{\alpha} \rightarrow \nu_{\beta}\right)= & \left|\left[\tilde{U} \exp (-i \mathcal{E} L) \tilde{U}^{-1}\right]_{\beta \alpha}\right|^{2} \\
= & \delta_{\alpha \beta}-4 \sum_{j<k} \operatorname{Re}\left(\tilde{X}_{j}^{\alpha \beta} \tilde{X}_{k}^{\alpha \beta *}\right) \sin ^{2}\left(\frac{\Delta \tilde{E}_{j k} L}{2}\right) \\
& -2 \sum_{j<k} \operatorname{Im}\left(\tilde{X}_{j}^{\alpha \beta} \tilde{X}_{k}^{\alpha \beta *}\right) \sin \left(\Delta \tilde{E}_{j k} L\right),
\end{aligned}
$$

where we have defined

$$
\begin{aligned}
\tilde{X}_{j}^{\alpha \beta} & \equiv \tilde{U}_{\alpha j} \tilde{U}_{\beta j}^{*}, \\
\Delta \tilde{E}_{j k} & \equiv \tilde{E}_{j}-\tilde{E}_{k},
\end{aligned}
$$

and $\alpha, \beta=(e, \mu, \tau)$ and $j, k=(1,2,3)$ stand for the indices of the flavor and mass eigenstates, respectively. Eq. (A5 shows that we can obtain the oscillation probability if we know the energy eigenvalues $\tilde{E}_{j}$ and $\tilde{X}_{j}^{\alpha \beta}$. The former can be obtained by the eigenvalue equation $\left|U \mathcal{E} U^{-1}+\mathcal{A}-t \mathbf{1}\right|=0$, while the latter can be obtained by the formalism of Kimura, Takamura and Yokomakura [68, 69].

Let us briefly see how we get $\tilde{X}_{j}^{\alpha \beta}[67-71]$. From the unitarity condition of the matrix $\tilde{U}$, we have

$$
\delta_{\alpha \beta}=\left[\tilde{U} \tilde{U}^{-1}\right]_{\alpha \beta}=\sum_{j} \tilde{U}_{\alpha j} \tilde{U}_{\beta j}^{*}=\sum_{j} \tilde{X}_{j}^{\alpha \beta}
$$

Note that the quantity $\tilde{X}_{j}^{\alpha \beta}$ was defined in Eq. (A6). Furthermore we take the $(\alpha, \beta)$ component of the both hand sides in Eq. (A4) and its square:

$$
\begin{array}{r}
{\left[U \mathcal{E} U^{-1}+\mathcal{A}\right]_{\alpha \beta}=\left[\tilde{U} \tilde{\mathcal{E}} \tilde{U}^{-1}\right]_{\alpha \beta}=\sum_{j} \tilde{U}_{\alpha j} \tilde{E}_{j} \tilde{U}_{\beta j}^{*}=\sum_{j} \tilde{E}_{j} \tilde{X}_{j}^{\alpha \beta}} \\
{\left[\left(U \mathcal{E} U^{-1}+\mathcal{A}\right)^{2}\right]_{\alpha \beta}=\left[\tilde{U} \tilde{\mathcal{E}}^{2} \tilde{U}^{-1}\right]_{\alpha \beta}=\sum_{j} \tilde{U}_{\alpha j} \tilde{E}_{j}^{2} \tilde{U}_{\beta j}^{*}=\sum_{j} \tilde{E}_{j}^{2} \tilde{X}_{j}^{\alpha \beta} .}
\end{array}
$$

From Eqs. (A7) and (A8), we have

$$
\left(\begin{array}{ccc}
1 & 1 & 1 \\
\tilde{E}_{1} & \tilde{E}_{2} & \tilde{E}_{3} \\
\tilde{E}_{1}^{2} & \tilde{E}_{2}^{2} & \tilde{E}_{3}^{2}
\end{array}\right)\left(\begin{array}{c}
\tilde{X}_{1}^{\alpha \beta} \\
\tilde{X}_{2}^{\alpha \beta} \\
\tilde{X}_{3}^{\alpha \beta}
\end{array}\right)=\left(\begin{array}{c}
Y_{1}^{\alpha \beta} \\
Y_{2}^{\alpha \beta} \\
Y_{3}^{\alpha \beta}
\end{array}\right),
$$

where we have introduced notations $\left(Y_{1}^{\alpha \beta}=\delta_{\alpha \beta}\right)$ :

$$
Y_{\ell}^{\alpha \beta} \equiv\left[\left(U \mathcal{E} U^{-1}+\mathcal{A}\right)^{\ell-1}\right]_{\alpha \beta} .
$$


Eq. (A9) can be easily solved by inverting the Vandermonde matrix:

$$
\left(\begin{array}{c}
\tilde{X}_{1}^{\alpha \beta} \\
\tilde{X}_{2}^{\alpha \beta} \\
\tilde{X}_{3}^{\alpha \beta}
\end{array}\right)=\left(\begin{array}{l}
\frac{1}{\Delta \tilde{E}_{21} \Delta \tilde{E}_{31}}\left(\tilde{E}_{2} \tilde{E}_{3},-\left(\tilde{E}_{2}+\tilde{E}_{3}\right), 1\right) \\
\frac{-1}{\Delta \tilde{E}_{21} \Delta \tilde{E}_{32}}\left(\tilde{E}_{3} \tilde{E}_{1},-\left(\tilde{E}_{3}+\tilde{E}_{1}\right), 1\right) \\
\frac{1}{\Delta \tilde{E}_{31} \Delta \tilde{E}_{32}}\left(\tilde{E}_{1} \tilde{E}_{2},-\left(\tilde{E}_{1}+\tilde{E}_{2}\right), 1\right)
\end{array}\right)\left(\begin{array}{c}
Y_{1}^{\alpha \beta} \\
Y_{2}^{\alpha \beta} \\
Y_{3}^{\alpha \beta}
\end{array}\right) .
$$

Note that $Y_{\ell}^{\alpha \beta}(\ell=1,2,3)$ in Eq. $(\underline{A 10})$ can be expressed by the known quantities, so that the oscillation probability (A5) can be expressed analytically in terms of the known quantities.

To summarize this appendix, the following is a set of the procedures to obtain the analytic form of the oscillation probability in the presence of constant generic matter potential:

(i) Obtain the roots $\tilde{E}_{j}$ of the eigenvalue equation $\left|U \mathcal{E} U^{-1}+\mathcal{A}-t \mathbf{1}\right|=0$.

(ii) Obtain the coefficients $\tilde{X}_{j}^{\alpha \beta}$ in Eq. (A11) by evaluating $Y_{\ell}^{\alpha \beta} \equiv\left[\left(U \mathcal{E} U^{-1}+\mathcal{A}\right)^{\ell-1}\right]_{\alpha \beta}$.

(iii) Substitute $\tilde{E}_{j}$ and $\tilde{X}_{j}^{\alpha \beta}$ in Eq. (A5) to obtain $P\left(\nu_{\alpha} \rightarrow \nu_{\beta}\right)$.

Appendix B: The disappearance oscillation probability of $\nu_{\mu}$ at high-energy in the standard three-flavor scenario in matter

In this appendix we derive the high-energy behavior of the the oscillation probability $P\left(\nu_{\mu} \rightarrow \nu_{\mu}\right)$ in the standard three-flavor scenario in matter. At high-energy we can safely ignore the contribution from the solar neutrino mass-squared difference $\Delta m_{21}^{2}$. In this case the three eigenvalues can be easily obtained as $\tilde{E}_{1}, \tilde{E}_{2}, \tilde{E}_{3}=\lambda_{-}, 0, \lambda_{+}[72]$, where

$$
\lambda_{ \pm} \equiv \frac{\Delta E_{31}+A}{2} \pm \frac{1}{2} \sqrt{\left(\Delta E_{31} \cos 2 \theta_{13}-A\right)^{2}+\left(\Delta E_{31} \sin 2 \theta_{13}\right)^{2}} .
$$

They have the following behavior at high-energy $\left(\left|\Delta E_{31}\right|=\left|\Delta m_{31}^{2}\right| / 2 E \ll A\right)$ :

$$
\begin{aligned}
& \tilde{E}_{1} \simeq \Delta E_{31} c_{13}^{2} \\
& \tilde{E}_{2}=0 \\
& \tilde{E}_{3} \simeq A,
\end{aligned}
$$

where $c_{j k} \equiv \cos \theta_{j k}$. From these and the fact $Y_{2}^{\mu \mu}=\Delta E_{31}\left|U_{\mu 3}\right|^{2}, Y_{3}^{\mu \mu}=\Delta E_{31}^{2}\left|U_{\mu 3}\right|^{2}$ we get the coefficients

$$
X_{1}^{\mu \mu} \simeq \frac{\left|U_{\mu 3}\right|^{2}}{c_{13}^{2}}, X_{2}^{\mu \mu} \simeq 1-\frac{\left|U_{\mu 3}\right|^{2}}{c_{13}^{2}}, X_{3}^{\mu \mu}=\left(\frac{\Delta E_{31}}{A}\right)^{2}\left|U_{\mu 3}\right|^{2} s_{13}^{2},
$$

where $s_{j k} \equiv \sin \theta_{j k}$. Hence we obtain the following behavior of the disappearance probability at high-energy:

$$
\begin{aligned}
\lim _{E \rightarrow \infty} \frac{1-P\left(\nu_{\mu} \rightarrow \nu_{\mu}\right)}{\left(\Delta E_{31} / A\right)^{2}} \simeq & \lim _{E \rightarrow \infty} \frac{1}{\left(\Delta E_{31} / A\right)^{2}}\left[4 \frac{\left|U_{\mu 3}\right|^{2}}{c_{13}^{2}}\left(1-\frac{\left|U_{\mu 3}\right|^{2}}{c_{13}^{2}}\right) \sin ^{2}\left(\frac{c_{13}^{2} \Delta E_{31} L}{2}\right)\right. \\
& \left.+4\left(\frac{\Delta E_{31}}{A}\right)^{2}\left|U_{\mu 3}\right|^{2} s_{13}^{2} \sin ^{2}\left(\frac{A L}{2}\right)\right] \\
\simeq & \sin ^{2} 2 \theta_{23}\left(\frac{c_{13}^{2} A L}{2}\right)^{2}+s_{23}^{2} \sin ^{2} 2 \theta_{13} \sin ^{2}\left(\frac{A L}{2}\right)
\end{aligned}
$$


or in other words, we have

$$
1-P\left(\nu_{\mu} \rightarrow \nu_{\mu}\right) \propto \frac{1}{E^{2}}
$$

Eq. ([6) is the prediction for the high-energy behavior of the disappearance probability from the standard three-flavor oscillations, and it is perfectly consistent with the atmospheric neutrino data [73].

\section{Appendix C: The disappearance oscillation probability of $\nu_{\mu}$ at high-energy with new physics: (i) $\epsilon_{e \mu}$ and $\epsilon_{\mu \tau}$}

In this appendix we consider the disappearance oscillation probability with new physics in propagation given by Eq. (2), particularly with nonvanishing $\epsilon_{e \mu}$ and $\epsilon_{\mu \tau}$. To simplify calculations, let us normalize $U \mathcal{E} U^{-1}+\mathcal{A}$ by $A\left(1+\epsilon_{e e}\right)$ :

$$
U \mathcal{E} U^{-1}+\mathcal{A}=A^{\prime}\left[U \operatorname{diag}\left(0, \frac{\Delta E_{21}}{A^{\prime}}, \frac{\Delta E_{31}}{A^{\prime}}\right) U^{-1}+\left(\begin{array}{ccc}
1 & \epsilon_{e \mu}^{\prime} & \epsilon_{e \tau}^{\prime} \\
\left(\epsilon_{e \mu}^{\prime}\right)^{*} & \epsilon_{\mu \mu}^{\prime} & \epsilon_{\mu \tau}^{\prime} \\
\left(\epsilon_{e \tau}^{\prime}\right)^{*} & \left(\epsilon_{\mu \tau}^{\prime}\right)^{*} & \epsilon_{\tau \tau}^{\prime}
\end{array}\right)\right]
$$

where we have defined 7

$$
\begin{aligned}
A^{\prime} & \equiv A\left(1+\epsilon_{e e}\right) \\
\epsilon_{\alpha \beta}^{\prime} & \equiv \frac{\epsilon_{\alpha \beta}}{1+\epsilon_{e e}} .
\end{aligned}
$$

From (3) we assume that $\left|\epsilon_{e \mu}^{\prime}\right|,\left|\epsilon_{\mu \mu}^{\prime}\right|,\left|\epsilon_{\mu \tau}^{\prime}\right|$ are relatively small compared with one, while $\left|\epsilon_{e \tau}^{\prime}\right|$ and $\left|\epsilon_{\tau \tau}^{\prime}\right|$ could be of order $\mathcal{O}(1)$.

First, we evaluate the energy eigenvalues for $\left|\Delta E_{31} / A^{\prime}\right| \ll 1,\left|\epsilon_{\alpha \mu}^{\prime}\right| \ll 1(\alpha=e, \mu, \tau)$, $\left.\left|\epsilon_{\tau \tau}^{\prime}-\right| \epsilon_{e \tau}^{\prime}\right|^{2} \mid \ll 1$, while $\left|\epsilon_{\tau \tau}^{\prime}\right|$ and $\left|\epsilon_{e \tau}^{\prime}\right|$ are not necessarily expected to be small. Furthermore, we will ignore small corrections due to $\Delta E_{21} / A^{\prime}$ and $\sin \theta_{13}$. Then the mass matrix becomes

$$
\begin{aligned}
& \frac{1}{A^{\prime}}\left(U \mathcal{E} U^{-1}+\mathcal{A}\right) \\
\simeq & \left(\begin{array}{ccc}
1 & \epsilon_{e \mu}^{\prime} & \epsilon_{e \tau}^{\prime} \\
\left(\epsilon_{\mu e}^{\prime}\right)^{*} & \epsilon_{\mu \mu}^{\prime}+s_{23}^{2} \eta & \epsilon_{\mu \tau}^{\prime}+c_{23} s_{23} \eta \\
\left(\epsilon_{\tau e}^{\prime}\right)^{*} & \left(\epsilon_{\mu \tau}^{\prime}\right)^{*}+c_{23} s_{23} \eta & \epsilon_{\tau \tau}^{\prime}+c_{23}^{2} \eta
\end{array}\right) \equiv\left(\begin{array}{ccc}
1 & \epsilon_{e \mu}^{\prime} & \epsilon_{e \tau}^{\prime} \\
\left(\epsilon_{e \mu}^{\prime}\right)^{*} & \epsilon_{\mu \mu}^{\prime \prime} & \epsilon_{\mu \tau}^{\prime \prime} \\
\left(\epsilon_{e \tau}^{\prime}\right)^{*} & \left(\epsilon_{\mu \tau}^{\prime \prime}\right)^{*} & \epsilon_{\tau \tau}^{\prime \prime}
\end{array}\right),
\end{aligned}
$$

where

$$
\eta \equiv \Delta E_{31} / A^{\prime}
$$

has been introduced and the difference between $\epsilon_{\alpha \beta}^{\prime \prime}$ and $\epsilon_{\alpha \beta}^{\prime}$ is the term proportional to $\eta$. Expanding the eigenvalue equation to first order in $\epsilon_{\tau \tau}^{\prime}-\left|\epsilon_{e \tau}^{\prime}\right|^{2}$, and to second order in $\eta$, $\epsilon_{e \mu}^{\prime}, \epsilon_{\mu \mu}^{\prime}$ and $\epsilon_{\mu \tau}^{\prime}$, we have

$$
\begin{aligned}
0= & \left|\frac{1}{A^{\prime}}\left(U \mathcal{E} U^{-1}+\mathcal{A}\right)-t \mathbf{1}\right| \\
= & -t^{3}+t^{2}\left(1+\epsilon_{\tau \tau}^{\prime}+\eta\right) \\
& +t\left\{\left|\epsilon_{e \mu}^{\prime}\right|^{2}+\left|\epsilon_{\mu \tau}^{\prime \prime}\right|^{2}+\left|\epsilon_{e \tau}^{\prime}\right|^{2}-\epsilon_{\tau \tau}^{\prime}-\left(1+s_{23}^{2} \epsilon_{\tau \tau}^{\prime}\right) \eta-c_{23}^{2} s_{23}^{2} \eta^{2}\right\} \\
& -\left[\left|\epsilon_{\mu \tau}^{\prime \prime}\right|^{2}+\epsilon_{\tau \tau}^{\prime}\left|\epsilon_{e \mu}^{\prime}\right|^{2}-2 \operatorname{Re}\left\{\epsilon_{e \mu}^{\prime} \epsilon_{\mu \tau}^{\prime \prime}\left(\epsilon_{e \tau}^{\prime}\right)^{*}\right\}+s_{23}^{2}\left(\left|\epsilon_{e \tau}^{\prime}\right|^{2}-\epsilon_{\tau \tau}^{\prime}\right) \eta-c_{23}^{2} s_{23}^{2} \eta^{2}\right] .
\end{aligned}
$$

7 Here, we have assumed that $\left|1+\epsilon_{e e}\right|$ is not very small. This assumption is reasonable, because otherwise the solar neutrino would not feel the matter effect very much and it would contradict with the solar neutrino data. 
In the limit where all small parameters $\eta, \epsilon_{\tau \tau}^{\prime}-\left|\epsilon_{e \tau}^{\prime}\right|^{2}, \epsilon_{e \mu}^{\prime}, \epsilon_{\mu \mu}^{\prime}, \epsilon_{\mu \tau}^{\prime}$, are zero, Eq. (C5) gives the roots $t=0,0,1+\epsilon_{\tau \tau}^{\prime}$. If we include the corrections to first order in the small parameters, then we get

$$
\begin{aligned}
\left\{\begin{array}{c}
t_{2} \\
t_{1}
\end{array}\right\}= & \frac{1+s_{23}^{2} \epsilon_{\tau \tau}^{\prime}}{1+\epsilon_{\tau \tau}^{\prime}} \frac{\eta}{2} \\
& \pm \frac{1}{2\left(1+\epsilon_{\tau \tau}^{\prime}\right)}\left[4 ( 1 + \epsilon _ { \tau \tau } ^ { \prime } ) \left\{\left|\epsilon_{\mu \tau}^{\prime \prime}\right|^{2}+\epsilon_{\tau \tau}^{\prime}\left|\epsilon_{e \mu}^{\prime}\right|^{2}-2 \operatorname{Re}\left(\epsilon_{e \mu}^{\prime} \epsilon_{\mu \tau}^{\prime \prime}\left(\epsilon_{e \tau}^{\prime}\right)^{*}\right)\right.\right. \\
& \left.\left.\quad+s_{23}^{2}\left(\left|\epsilon_{e \tau}^{\prime}\right|^{2}-\epsilon_{\tau \tau}^{\prime}\right) \eta-c_{23}^{2} s_{23}^{2} \eta^{2}\right\}+\left(1+s_{23}^{2} \epsilon_{\tau \tau}^{\prime}\right)^{2} \eta^{2}\right]^{1 / 2} \\
t_{3}=1 & +\epsilon_{\tau \tau}^{\prime}+\mathcal{O}\left(\eta, \epsilon_{\alpha \mu}^{\prime}\right) .
\end{aligned}
$$

Next, we evaluate the quantities $Y_{j}^{\mu \mu}$. They are given by

$$
\begin{aligned}
Y_{2}^{\mu \mu} & =\frac{1}{A^{\prime}}\left(U \mathcal{E} U^{-1}+\mathcal{A}\right)_{\mu \mu}=\epsilon_{\mu \mu}^{\prime}+s_{23}^{2} \eta=\epsilon_{\mu \mu}^{\prime \prime}, \\
Y_{3}^{\mu \mu} & =\frac{1}{\left(A^{\prime}\right)^{2}}\left[\left(U \mathcal{E} U^{-1}+\mathcal{A}\right)^{2}\right]_{\mu \mu}=\left|\epsilon_{e \mu}^{\prime}\right|^{2}+\left|\epsilon_{\mu \mu}^{\prime \prime}\right|^{2}+\left|\epsilon_{\mu \tau}^{\prime \prime}\right|^{2},
\end{aligned}
$$

where $\epsilon_{\alpha \beta}^{\prime \prime}$ are defined in Eq. (C3). Plugging these results into Eq. (A11), we obtain

$$
\begin{aligned}
& \tilde{X}_{1}^{\mu \mu}=\frac{1}{\Delta t_{21}\left(1+\epsilon_{\tau \tau}^{\prime}\right)}\left[\left(t_{2}-\epsilon_{\mu \mu}^{\prime}-s_{23}^{2} \eta\right)\left(1+\epsilon_{\tau \tau}^{\prime}\right)+\left|\epsilon_{e \mu}^{\prime}\right|^{2}+\left|\epsilon_{\mu \mu}^{\prime \prime}\right|^{2}+\left|\epsilon_{\mu \tau}^{\prime \prime}\right|^{2}\right] \\
& \tilde{X}_{2}^{\mu \mu}=\frac{-1}{\Delta t_{21}\left(1+\epsilon_{\tau \tau}^{\prime}\right)}\left[\left(t_{1}-\epsilon_{\mu \mu}^{\prime}-s_{23}^{2} \eta\right)\left(1+\epsilon_{\tau \tau}^{\prime}\right)+\left|\epsilon_{e \mu}^{\prime}\right|^{2}+\left|\epsilon_{\mu \mu}^{\prime \prime}\right|^{2}+\left|\epsilon_{\mu \tau}^{\prime \prime}\right|^{2}\right] \\
& \tilde{X}_{3}^{\mu \mu}=\frac{1}{\left(1+\epsilon_{\tau \tau}^{\prime}\right)^{2}}\left[t_{1} t_{2}-\frac{1+s_{23}^{2} \epsilon_{\tau \tau}^{\prime}}{1+\epsilon_{\tau \tau}^{\prime}}\left(\epsilon_{\mu \mu}^{\prime}+s_{23}^{2} \eta\right) \eta+\left|\epsilon_{e \mu}^{\prime}\right|^{2}+\left|\epsilon_{\mu \mu}^{\prime \prime}\right|^{2}+\left|\epsilon_{\mu \tau}^{\prime \prime}\right|^{2}\right]
\end{aligned}
$$

where $\Delta t_{21} \equiv t_{2}-t_{1}$ and $t_{j}(j=1,2,3)$ are given by Eq. (C6).

Let us now consider the situation in which the limit $E \rightarrow \infty$ or $\eta=\Delta m_{31}^{2} /\left(2 A^{\prime} E\right) \rightarrow 0$ is taken while $\epsilon_{e \mu}^{\prime} \neq 0$ and $\epsilon_{\mu \tau}^{\prime} \neq 0$ are kept. In this case, from Eq. (3), which implies $\left|\epsilon_{e \mu}^{\prime}\right|^{2} \lesssim 0.1$ and $\left|\epsilon_{\mu \tau}^{\prime}\right|^{2} \lesssim 0.1$, and from Eqs. (다), we get

$$
\begin{aligned}
& 1-P\left(\nu_{\mu} \rightarrow \nu_{\mu}\right) \\
\simeq & 4 \tilde{X}_{1}^{\mu \mu} \tilde{X}_{2}^{\mu \mu} \sin ^{2}\left(\frac{\Delta t_{21} A^{\prime} L}{2}\right)+4 \tilde{X}_{3}^{\mu \mu}\left(1-\tilde{X}_{3}^{\mu \mu}\right) \sin ^{2}\left(\frac{\left(1+\epsilon_{\tau \tau}^{\prime}\right) A^{\prime} L}{2}\right),
\end{aligned}
$$

where

$$
\begin{aligned}
4 \tilde{X}_{1}^{\mu \mu} \tilde{X}_{2}^{\mu \mu} & \simeq 1-\frac{1+\epsilon_{\tau \tau}^{\prime}}{\left|\epsilon_{\mu \tau}^{\prime}\right|^{2}+\epsilon_{\tau \tau}^{\prime}\left|\epsilon_{e \mu}^{\prime}\right|^{2}-2 \operatorname{Re}\left(\epsilon_{e \mu}^{\prime} \epsilon_{\mu \tau}^{\prime}\left(\epsilon_{e \tau}^{\prime}\right)^{*}\right)}\left(\frac{\left|\epsilon_{e \mu}^{\prime}\right|^{2}+\left|\epsilon_{\mu \mu}^{\prime}\right|^{2}+\left|\epsilon_{\mu \tau}^{\prime}\right|^{2}}{1+\epsilon_{\tau \tau}^{\prime}}-\epsilon_{\mu \mu}^{\prime}\right)^{2}, \\
4 \tilde{X}_{3}^{\mu \mu}\left(1-\tilde{X}_{3}^{\mu \mu}\right) & \simeq 4 \tilde{X}_{3}^{\mu \mu} \\
& \simeq 4 \frac{\left|\epsilon_{e \mu}^{\prime}\right|^{2}+\left|\epsilon_{\mu \mu}^{\prime}\right|^{2}+\left|\epsilon_{\mu \tau}^{\prime}\right|^{2}}{\left(1+\epsilon_{\tau \tau}^{\prime}\right)^{2}}-4 \frac{\left|\epsilon_{\mu \tau}^{\prime}\right|^{2}+\epsilon_{\tau \tau}^{\prime}\left|\epsilon_{e \mu}^{\prime}\right|^{2}-2 \operatorname{Re}\left(\epsilon_{e \mu}^{\prime} \epsilon_{\mu \tau}^{\prime}\left(\epsilon_{e \tau}^{\prime}\right)^{*}\right)}{\left(1+\epsilon_{\tau \tau}^{\prime}\right)^{3}}, \\
\Delta t_{21} & \simeq 2 t_{2} \simeq 2\left[\frac{\left|\epsilon_{\mu \tau}^{\prime}\right|^{2}+\epsilon_{\tau \tau}^{\prime}\left|\epsilon_{e \mu}^{\prime}\right|^{2}-2 \operatorname{Re}\left(\epsilon_{e \mu}^{\prime} \epsilon_{\mu \tau}^{\prime}\left(\epsilon_{e \tau}^{\prime}\right)^{*}\right)}{1+\epsilon_{\tau \tau}^{\prime}}\right] .
\end{aligned}
$$

Recovering the original notations, we obtain Eq. (8) from Eq. (C8). Since the argument $\left(1+\epsilon_{\tau \tau}^{\prime}\right) A^{\prime} L / 2=\left(1+\epsilon_{e e}+\epsilon_{\tau \tau}\right) A L / 2$ of the sine function of the second term on the right 
hand side of Eq. (8) is of the same order as $A L / 2$, in order for Eq. (8) to be consistent with the atmospheric neutrino data at high-energy (cf. Eq. (B1)), at least either of the followings has to be satisfied: (i) $\Delta t_{21}=\tilde{X}_{3}^{\mu \mu}=0$ or (ii) $4 \tilde{X}_{1}^{\mu \mu} \tilde{X}_{2}^{\mu \mu}=\tilde{X}_{3}^{\mu \mu}=0$. Let us introduce the two quantities:

$$
\begin{aligned}
& F \equiv\left|\epsilon_{e \mu}^{\prime}\right|^{2}+\left|\epsilon_{\mu \mu}^{\prime}\right|^{2}+\left|\epsilon_{\mu \tau}^{\prime}\right|^{2} \\
& G \equiv\left|\epsilon_{\mu \tau}^{\prime}\right|^{2}+\epsilon_{\tau \tau}^{\prime}\left|\epsilon_{e \mu}^{\prime}\right|^{2}-2 \operatorname{Re}\left(\epsilon_{e \mu}^{\prime} \epsilon_{\mu \tau}^{\prime}\left(\epsilon_{e \tau}^{\prime}\right)^{*}\right) .
\end{aligned}
$$

Then, in the case of (i), we have $F \simeq G \simeq 0$, which implies $\left|\epsilon_{e \mu}^{\prime}\right| \simeq\left|\epsilon_{\mu \mu}^{\prime}\right| \simeq\left|\epsilon_{\mu \tau}^{\prime}\right| \simeq 0$. In the case of (ii), on the other hand, we get

$$
\begin{aligned}
\left(\frac{F}{1+\epsilon_{\tau \tau}^{\prime}}-\epsilon_{\mu \mu}^{\prime}\right)^{2} & \simeq \frac{G}{1+\epsilon_{\tau \tau}^{\prime}} \\
F & \simeq \frac{G}{1+\epsilon_{\tau \tau}^{\prime}}
\end{aligned}
$$

From these equations and the fact that $\left|\epsilon_{e \mu}^{\prime}\right|^{2} \lesssim 0.1$ and $\left|\epsilon_{\mu \tau}^{\prime}\right|^{2} \lesssim 0.1$, it follows that

$$
\left|\epsilon_{e \mu}^{\prime}\right|^{2}+\left|\epsilon_{\mu \tau}^{\prime}\right|^{2} \simeq \epsilon_{\tau \tau}^{\prime}\left(\epsilon_{\mu \mu}^{\prime}\right)^{2}
$$

i.e., in the original notation,

$$
\left|\epsilon_{e \mu}\right|^{2}+\left|\epsilon_{\mu \tau}\right|^{2} \simeq \frac{\epsilon_{\tau \tau}\left(\epsilon_{\mu \mu}\right)^{2}}{1+\epsilon_{e e}}
$$

has to be satisfied at least, if a consistent solution exists at all. The bounds from (i) or (ii) are stronger that of Eq. (3), and it is expected that the three parameters $\epsilon_{e \mu}, \epsilon_{\mu \mu}, \epsilon_{\mu \tau}$ are negligibly small compared to $\epsilon_{e e}, \epsilon_{e \tau}$ and $\epsilon_{\tau \tau}$, although this speculation has to be verified by numerical calculations.

Appendix D: The disappearance oscillation probability of $\nu_{\mu}$ at high-energy with new physics: (ii) $\epsilon_{\tau \tau}-\left|\epsilon_{e \tau}\right|^{2} /\left(1+\epsilon_{e e}\right)$

In this appendix we assume that $\epsilon_{e \mu}$ and $\epsilon_{\mu \tau}$ are zero and discuss the case in which $\epsilon_{e e}$, $\epsilon_{e \tau}$ and $\epsilon_{\tau \tau}$ are of order $\mathcal{O}(1)$. In this case the energy eigenvalue equation (C5) becomes

$$
0=-t^{3}+t^{2}\left(1+\epsilon_{\tau \tau}^{\prime}+\eta\right)-t\left\{\zeta^{\prime}+\left(1+s_{23}^{2} \epsilon_{\tau \tau}^{\prime}\right) \eta\right\}+s_{23}^{2} \zeta^{\prime} \eta
$$

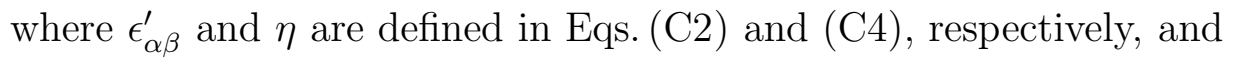

$$
\zeta^{\prime} \equiv \epsilon_{\tau \tau}^{\prime}-\left|\epsilon_{e \tau}^{\prime}\right|^{2}
$$

In the case where $|\eta| \ll 1$, the three roots are given by

$$
\begin{aligned}
& t_{1} \simeq 0 \\
&\left\{\begin{array}{l}
t_{2} \\
t_{3}
\end{array}\right\} \simeq \frac{1+\epsilon_{\tau \tau}^{\prime}}{2} \mp \sqrt{\left(\frac{1+\epsilon_{\tau \tau}^{\prime}}{2}\right)^{2}-\zeta^{\prime}}
\end{aligned}
$$


and $\tilde{X}_{j}^{\mu \mu}$ are given by

$$
\begin{aligned}
& \tilde{X}_{1}^{\mu \mu} \simeq 1-\frac{1+\epsilon_{\tau \tau}^{\prime}}{\zeta^{\prime}} \eta s_{23}^{2} \\
& \tilde{X}_{2}^{\mu \mu} \simeq \frac{t_{3}}{t_{2} \Delta t_{32}} \eta s_{23}^{2}=\frac{t_{3}^{2}}{\zeta^{\prime} \Delta t_{32}} \eta s_{23}^{2} \\
& \tilde{X}_{3}^{\mu \mu} \simeq-\frac{t_{2}}{t_{3} \Delta t_{32}} \eta s_{23}^{2}=-\frac{\zeta^{\prime}}{t_{3}^{2} \Delta t_{32}} \eta s_{23}^{2} .
\end{aligned}
$$

The disappearance oscillation probability has the following behavior:

$$
\begin{aligned}
1-P\left(\nu_{\mu} \rightarrow \nu_{\mu}\right) \simeq & -\eta \frac{4 s_{23}^{2}}{\Delta t_{32}}\left[\frac{t_{3}^{2}}{\zeta^{\prime}} \sin ^{2}\left(\frac{\zeta^{\prime} A^{\prime} L}{2 t_{3}}\right)+\frac{\zeta^{\prime}}{t_{3}^{2}} \sin ^{2}\left(\frac{t_{3} A^{\prime} L}{2}\right)\right] \\
& +\eta^{2} \frac{4 s_{23}^{4}}{\left(\Delta t_{32}\right)^{2}} \sin ^{2}\left(\frac{A^{\prime} \Delta t_{32} L}{2}\right)
\end{aligned}
$$

where $\Delta t_{32} \equiv t_{3}-t_{2}$. By recovering the original notations, we obtain Eq. (9).

Appendix E: The disappearance oscillation probability of $\nu_{\mu}$ at high-energy with new physics: (iii) $\epsilon_{e e}, \epsilon_{e \tau}$ and $\epsilon_{\tau \tau}=\left|\epsilon_{e \tau}\right|^{2} /\left(1+\epsilon_{e e}\right)$

In this appendix we assume $\zeta^{\prime}=\epsilon_{\tau \tau}^{\prime}-\left|\epsilon_{e \tau}^{\prime}\right|^{2}=0$ and derive the high-energy behavior (11). In this case, introducing the new variable $t_{\beta} \equiv\left|\epsilon_{e \tau}\right| /\left(1+\epsilon_{e e}\right)$ (cf. Eq. (10)), we have

$$
\begin{aligned}
\left|\epsilon_{e \tau}^{\prime}\right| & =\frac{\left|\epsilon_{e \tau}\right|}{1+\epsilon_{e e}}=t_{\beta}, \\
\epsilon_{\tau \tau}^{\prime} & =\left|\epsilon_{e \tau}^{\prime}\right|^{2}=t_{\beta}^{2} .
\end{aligned}
$$

Recovering the small corrections due to $\theta_{13}$, we have the energy eigenvalue equation:

$$
\begin{aligned}
0 & =-t^{3}+t^{2}\left(1+t_{\beta}^{2}+\eta\right)-t \eta\left(1+t_{\beta}^{2}-\left|U_{e 3}+U_{\tau 3} \epsilon_{e \tau}^{\prime}\right|^{2}\right) \\
& =-t\left\{t^{2}-\left(1 / c_{\beta}^{2}+\eta\right) t+\eta\left(c_{13}^{\prime \prime}\right)^{2} / c_{\beta}^{2}\right\}
\end{aligned}
$$

where $c_{13}^{\prime \prime} \equiv \cos \theta_{13}^{\prime \prime}$ and $\theta_{13}^{\prime \prime}$ is defined by Eq. (F6) The three roots in this case are $t_{1}=0$, $t_{2} \simeq \eta\left(c_{13}^{\prime \prime}\right)^{2}, t_{3} \simeq 1 / c_{\beta}^{2}$. Then we have the following high-energy behavior (11) in the limit $E \rightarrow \infty$ :

$$
\begin{aligned}
\frac{1-P\left(\nu_{\mu} \rightarrow \nu_{\mu}\right)}{\left(\Delta E_{31} / A\right)^{2}}= & \frac{1-P\left(\nu_{\mu} \rightarrow \nu_{\mu}\right)}{\left(1+\epsilon_{e e}\right)^{2} \eta^{2}} \\
\simeq & 4 \frac{s_{23}^{2}}{\left(c_{13}^{\prime \prime}\right)^{2}}\left\{1-\frac{s_{23}^{2}}{\left(c_{13}^{\prime \prime}\right)^{2}}\right\}\left\{\frac{\left(c_{13}^{\prime \prime}\right)^{2} A L}{2}\right\}^{2} \\
& +\frac{s_{23}^{2}}{\left(c_{13}^{\prime \prime}\right)^{2}} \sin ^{2} 2 \theta_{13}^{\prime \prime}\left(\frac{c_{\beta}^{2}}{1+\epsilon_{e e}}\right)^{2} \sin ^{2}\left(\frac{\left(1+\epsilon_{e e}\right) A L}{2 c_{\beta}^{2}}\right) .
\end{aligned}
$$




\section{Appendix F: The analytic expression of $P\left(\nu_{\mu} \rightarrow \nu_{e}\right)$ with new physics in propagation}

In this appendix we discuss the analytic form of the oscillation probability $P\left(\nu_{\mu} \rightarrow \nu_{e}\right)$. From the formula (A5) we have

$$
\begin{aligned}
P\left(\nu_{\mu} \rightarrow \nu_{e}\right)= & -4 \operatorname{Re}\left(\tilde{X}_{1}^{\mu e} \tilde{X}_{2}^{\mu e *}\right) \sin ^{2}\left(\frac{\Delta \tilde{E}_{21} L}{2}\right)-4 \operatorname{Re}\left(\tilde{X}_{2}^{\mu e} \tilde{X}_{3}^{\mu e *}\right) \sin ^{2}\left(\frac{\Delta \tilde{E}_{32} L}{2}\right) \\
& -4 \operatorname{Re}\left(\tilde{X}_{1}^{\mu e} \tilde{X}_{3}^{\mu e *}\right) \sin ^{2}\left(\frac{\Delta \tilde{E}_{31} L}{2}\right) \\
& -8 \operatorname{Im}\left(\tilde{X}_{1}^{\mu e} \tilde{X}_{2}^{\mu e *}\right) \sin \left(\frac{\Delta \tilde{E}_{21} L}{2}\right) \sin \left(\frac{\Delta \tilde{E}_{32} L}{2}\right) \sin \left(\frac{\Delta \tilde{E}_{31} L}{2}\right) .
\end{aligned}
$$

From Eq. (A11) $\tilde{X}_{j}^{\mu e}$ can be expressed as

$$
\begin{aligned}
& \tilde{X}_{1}^{\mu e}=\frac{1}{\Delta \tilde{E}_{21} \Delta \tilde{E}_{31}}\left\{Y_{3}^{\mu e}-\left(\tilde{E}_{2}+\tilde{E}_{3}\right) Y_{2}^{\mu e}\right\} \\
& \tilde{X}_{2}^{\mu e}=\frac{-1}{\Delta \tilde{E}_{21} \Delta \tilde{E}_{32}}\left\{Y_{3}^{\mu e}-\left(\tilde{E}_{3}+\tilde{E}_{1}\right) Y_{2}^{\mu e}\right\} \\
& \tilde{X}_{3}^{\mu e}=\frac{1}{\Delta \tilde{E}_{31} \Delta \tilde{E}_{32}}\left\{Y_{3}^{\mu e}-\left(\tilde{E}_{1}+\tilde{E}_{2}\right) Y_{2}^{\mu e}\right\},
\end{aligned}
$$

where $Y_{j}^{\mu e}(j=2,3)$ is defined by Eq. (A10). and are given by

$$
\begin{aligned}
Y_{2}^{\mu e}= & \Delta E_{31} X_{3}^{\mu e}+\Delta E_{21} X_{2}^{\mu e} \\
Y_{3}^{\mu e}= & \left(\Delta E_{31}\right)^{2} X_{3}^{\mu e}+\left(\Delta E_{21}\right)^{2} X_{2}^{\mu e}+A \Delta E_{31}\left\{\left(1+\epsilon_{e e}\right) X_{3}^{\mu e}+\epsilon_{\tau e} X_{3}^{\mu \tau}\right\} \\
& +A \Delta E_{21}\left\{\left(1+\epsilon_{e e}\right) X_{2}^{\mu e}+\epsilon_{\tau e} X_{2}^{\mu \tau}\right\} .
\end{aligned}
$$

Here we have introduced the same quantity in vacuum:

$$
X_{j}^{\alpha \beta} \equiv U_{\alpha j} U_{\beta j}^{*}
$$

As shown in Ref. [67], in the limit $\Delta m_{21}^{2} \rightarrow 0, \tilde{E}_{j}$ can be expressed as roots of a quadratic equation. First, let us review how to obtain them. With the ansatz (16), we have

$$
\mathcal{A}=A^{\prime \prime} e^{i \gamma \lambda_{9}} e^{-i \beta \lambda_{5}} \frac{\lambda_{0}+\lambda_{9}}{2} e^{i \beta \lambda_{5}} e^{-i \gamma \lambda_{9}},
$$

where $\beta$ is defined by Eq. (10),

$$
\begin{aligned}
A^{\prime \prime} & \equiv A\left(1+\epsilon_{e e}\right) / c_{\beta}^{2}, \\
\gamma & \equiv \frac{1}{2} \arg \left(\epsilon_{e \tau}\right),
\end{aligned}
$$

and we have introduced notations for $3 \times 3$ hermitian matrices:

$$
\begin{array}{rlrl}
\lambda_{2} & \equiv\left(\begin{array}{ccc}
0 & -i & 0 \\
i & 0 & 0 \\
0 & 0 & 0
\end{array}\right), & \lambda_{5} \equiv\left(\begin{array}{ccc}
0 & 0 & -i \\
0 & 0 & 0 \\
i & 0 & 0
\end{array}\right), & \lambda_{7} \equiv\left(\begin{array}{ccc}
0 & 0 & 0 \\
0 & 0 & -i \\
0 & i & 0
\end{array}\right), \\
\lambda_{0} \equiv\left(\begin{array}{lll}
1 & 0 & 0 \\
0 & 0 & 0 \\
0 & 0 & 1
\end{array}\right), & \lambda_{9} \equiv\left(\begin{array}{ccc}
1 & 0 & 0 \\
0 & 0 & 0 \\
0 & 0 & -1
\end{array}\right) .
\end{array}
$$


Here, $\lambda_{2}, \lambda_{5}$ and $\lambda_{7}$ are the standard Gell-Mann matrices whereas $\lambda_{0}$ and $\lambda_{9}$ are the notations which are defined only in this paper.

The mass matrix can be written as

$$
U \mathcal{E} U^{-1}+\mathcal{A}=e^{i \gamma \lambda_{9}} e^{-i \beta \lambda_{5}}\left[e^{i \beta \lambda_{5}} e^{-i \gamma \lambda_{9}} U \mathcal{E} U^{-1} e^{i \gamma \lambda_{9}} e^{-i \beta \lambda_{5}}+\operatorname{diag}\left(A^{\prime \prime}, 0,0\right)\right] e^{i \beta \lambda_{5}} e^{-i \gamma \lambda_{9}} .
$$

Here, we introduce the two unitary matrices:

$$
\begin{aligned}
U^{\prime} & \equiv e^{i \beta \lambda_{5}} e^{-i \gamma \lambda_{9}} U \\
& \equiv \operatorname{diag}\left(1,1, e^{i \arg U_{\tau 3}^{\prime}}\right) U^{\prime \prime} \operatorname{diag}\left(e^{i \arg U_{e 1}^{\prime}}, e^{i \arg U_{e 2}^{\prime}}, 1\right),
\end{aligned}
$$

where $U$ is the $3 \times 3$ MNS matrix in the standard parametrization [1] and $U^{\prime \prime}$ was defined in the second line in such a way that the elements $U_{e 1}^{\prime \prime}, U_{e 2}^{\prime \prime}, U_{\tau 3}^{\prime \prime}$ be real to be consistent with the standard parametrization in Ref. 11] 8. Then we have

$$
\begin{aligned}
U \mathcal{E} U^{-1}+\mathcal{A}= & e^{i \gamma \lambda_{9}} e^{-i \beta \lambda_{5}} \operatorname{diag}\left(1,1, e^{i \arg U_{\tau 3}^{\prime}}\right)\left[U^{\prime \prime} \mathcal{E} U^{\prime \prime-1}+\operatorname{diag}\left(A^{\prime \prime}, 0,0\right)\right] \\
& \times \operatorname{diag}\left(1,1, e^{-i \arg U_{\tau 3}^{\prime}}\right) e^{i \beta \lambda_{5}} e^{-i \gamma \lambda_{9}}
\end{aligned}
$$

Before proceeding further, let us obtain the expression for the three mixing angles $\theta_{j k}^{\prime \prime}$ and the Dirac phase $\delta^{\prime \prime}$ in $U^{\prime \prime}$. Since

$$
U^{\prime}=\left(\begin{array}{ccc}
c_{\beta} e^{-i \gamma} U_{e 1}+s_{\beta} e^{i \gamma} U_{\tau 1} & c_{\beta} e^{-i \gamma} U_{e 2}+s_{\beta} e^{i \gamma} U_{\tau 2} & c_{\beta} e^{-i \gamma} U_{e 3}+s_{\beta} e^{i \gamma} U_{\tau 3} \\
U_{\mu 1} & U_{\mu 2} & U_{\mu 3} \\
c_{\beta} e^{-i \gamma} U_{\tau 1}-s_{\beta} e^{i \gamma} U_{e 1} & c_{\beta} e^{-i \gamma} U_{\tau 2}-s_{\beta} e^{i \gamma} U_{e 2} & c_{\beta} e^{-i \gamma} U_{\tau 3}-s_{\beta} e^{i \gamma} U_{e 3}
\end{array}\right),
$$

where $c_{\beta} \equiv \cos \beta, s_{\beta} \equiv \sin \beta$, we get

$$
\begin{aligned}
& \theta_{13}^{\prime \prime}=\sin ^{-1}\left|U_{e 3}^{\prime \prime}\right|=\sin ^{-1}\left|c_{\beta} e^{-i \gamma} U_{e 3}+s_{\beta} e^{i \gamma} U_{\tau 3}\right| \\
& \theta_{12}^{\prime \prime}=\tan ^{-1}\left(U_{e 2}^{\prime \prime} / U_{e 1}^{\prime \prime}\right)=\tan ^{-1}\left(\left|c_{\beta} e^{-i \gamma} U_{e 2}+s_{\beta} e^{i \gamma} U_{\tau 2}\right| /\left|c_{\beta} e^{-i \gamma} U_{e 1}+s_{\beta} e^{i \gamma} U_{\tau 1}\right|\right) \\
& \theta_{23}^{\prime \prime}=\tan ^{-1}\left(U_{\mu 3}^{\prime \prime} / U_{\tau 3}^{\prime \prime}\right)=\tan ^{-1}\left(U_{\mu 3} /\left|c_{\beta} e^{-i \gamma} U_{\tau 3}-s_{\beta} e^{i \gamma} U_{e 3}\right|\right) \\
& \delta^{\prime \prime}=-\arg \left[\left(U_{e 3}^{\prime}\right)^{-1} U_{e 1}^{\prime} U_{e 2}^{\prime} U_{\tau 3}^{\prime}\right] .
\end{aligned}
$$

As shown in Ref. [72], in the limit $\Delta m_{21}^{2} \rightarrow 0$, the matrix on the right hand side of Eq. (F5) can be diagonalized as follows:

$$
\begin{aligned}
& U^{\prime \prime} \mathcal{E} U^{\prime \prime-1}+\operatorname{diag}\left(A^{\prime \prime}, 0,0\right)-E_{1} \mathbf{1} \\
= & e^{i \theta_{23}^{\prime \prime} \lambda_{7}} \Gamma_{\delta^{\prime \prime}} e^{i \theta_{13}^{\prime \prime} \lambda_{5}} \Gamma_{\delta^{\prime \prime}}^{-1} e^{i \theta_{12}^{\prime \prime} \lambda_{2}} \operatorname{diag}\left(0,0, \Delta E_{31}\right) e^{-i \theta_{12}^{\prime \prime} \lambda_{2}} \Gamma_{\delta^{\prime \prime}} e^{-i \theta_{13}^{\prime \prime} \lambda_{5}} \Gamma_{\delta^{\prime \prime}}^{-1} e^{-i \theta_{23}^{\prime \prime} \lambda_{7}}+\operatorname{diag}\left(A^{\prime \prime}, 0,0\right) \\
= & e^{i \theta_{23}^{\prime \prime} \lambda_{7}} \Gamma_{\delta^{\prime \prime}}\left[e^{i \theta_{13}^{\prime \prime} \lambda_{5}} \operatorname{diag}\left(0,0, \Delta E_{31}\right) e^{-i \theta_{13}^{\prime \prime} \lambda_{5}}+\operatorname{diag}\left(A^{\prime \prime}, 0,0\right)\right] \Gamma_{\delta^{\prime \prime}}^{-1} e^{-i \theta_{23}^{\prime \prime} \lambda_{7}} \\
= & e^{i \theta_{23}^{\prime \prime} \lambda_{7}} \Gamma_{\delta^{\prime \prime}} e^{i \tilde{\theta}_{13}^{\prime \prime} \lambda_{5}} \operatorname{diag}\left(\Lambda_{-}, 0, \Lambda_{+}\right) e^{-i \tilde{\theta}_{13}^{\prime \prime} \lambda_{5}} \Gamma_{\delta^{\prime \prime}}^{-1} e^{-i \theta_{23}^{\prime \prime} \lambda_{7}},
\end{aligned}
$$

where $\Gamma_{\delta^{\prime \prime}} \equiv \operatorname{diag}\left(1,1, e^{-i \delta^{\prime \prime}}\right), \Delta E_{31} \equiv \Delta m_{31}^{2} / 2 E$, we have used the standard parametrization [1] $U^{\prime \prime} \equiv e^{i \theta_{23}^{\prime \prime} \lambda_{7}} \Gamma_{\delta^{\prime \prime}} e^{i \theta_{13}^{\prime \prime} \lambda_{5}} \Gamma_{\delta^{\prime \prime}}^{-1} e^{i \theta_{12}^{\prime \prime} \lambda_{2}}$, and the eigenvalues $\Lambda_{ \pm}$and the effective mixing angle $\tilde{\theta}_{13}^{\prime \prime}$ are defined by

$$
\begin{aligned}
\Lambda_{ \pm} & =\frac{1}{2}\left(\Delta E_{31}+A^{\prime \prime}\right) \pm \frac{1}{2} \sqrt{\left(\Delta E_{31} \cos 2 \theta_{13}^{\prime \prime}-A^{\prime \prime}\right)^{2}+\left(\Delta E_{31} \sin 2 \theta_{13}^{\prime \prime}\right)^{2}} \\
\tan 2 \tilde{\theta}_{13}^{\prime \prime} & =\frac{\Delta E_{31} \sin 2 \theta_{13}^{\prime \prime}}{\Delta E_{31} \cos 2 \theta_{13}^{\prime \prime}-A^{\prime \prime}} .
\end{aligned}
$$

8 The element $U_{\tau 2}^{\prime \prime}$ has to be also real, but it is already satisfied because $U_{\tau 2}^{\prime \prime}=U_{\tau 2}$. 
In the present case, since we consider the oscillation probability $P\left(\nu_{\mu} \rightarrow \nu_{e}\right)$ in Korea at low energy, i.e., at $L=1050 \mathrm{~km}$ with $E=0.7 \mathrm{GeV}$, the mass-squared difference due to the solar neutrino oscillation gives a non-negligible contribution $\Delta E_{21} L \sim 0.3$ and the correction in $\left|\Delta m_{21}^{2}\right| /\left|\Delta m_{31}^{2}\right|$ becomes important.

The discussions in Ref. [67] can be generalized to the case with non-vanishing $\Delta m_{21}^{2}$, since discussions up to Eq. (F5) are valid for a generic value of $\Delta m_{21}^{2} \neq 0$ and all we have to do is to obtain the correction to the energy eigenvalues due to small $\Delta m_{21}^{2}$. The energy eigenvalue $\tilde{E}_{j}$ to first order in $\left|\Delta m_{21}^{2}\right| /\left|\Delta m_{31}^{2}\right|$ can be computed and are given by

$$
\begin{aligned}
& \tilde{E}_{1}=\Lambda_{-}+\frac{\left(s_{12}^{\prime \prime}\right)^{2}}{\Lambda_{+}-\Lambda_{-}}\left\{\Lambda_{+}-\left(c_{13}^{\prime \prime}\right)^{2} A^{\prime \prime}\right\} \Delta E_{21} \\
& \tilde{E}_{2}=\left(c_{12}^{\prime \prime}\right)^{2} \Delta E_{21} \\
& \tilde{E}_{3}=\Lambda_{+}-\frac{\left(s_{12}^{\prime \prime}\right)^{2}}{\Lambda_{+}-\Lambda_{-}}\left\{\Lambda_{-}-\left(c_{13}^{\prime \prime}\right)^{2} A^{\prime \prime}\right\} \Delta E_{21},
\end{aligned}
$$

where $c_{j k}^{\prime \prime} \equiv \cos \theta_{j k}^{\prime \prime}$ and $s_{j k}^{\prime \prime} \equiv \sin \theta_{j k}^{\prime \prime}$. As seen from these expressions, in the limit $\Delta m_{21}^{2} \rightarrow 0$, all the quantities depend on the phase only through the combination $\delta+\arg \left(\epsilon_{e \tau}\right)$, since they depend only on $\theta_{13}^{\prime \prime}$ which is a function of the combination $\delta+\arg \left(\epsilon_{e \tau}\right)$. On the other hand, the oscillation probability $P\left(\nu_{\mu} \rightarrow \nu_{e}\right)$ in Korea has the moderate dependence on $\delta$ and $\arg \left(\epsilon_{e \tau}\right)$ separately, because the first order corrections in $\left|\Delta m_{21}^{2}\right| /\left|\Delta m_{31}^{2}\right|$ to $\tilde{E}_{j}$ have dependence on $\theta_{12}^{\prime \prime}$, which is approximately a function of $\arg \left(\epsilon_{e \tau}\right)$ only.

\section{Appendix G: The T violating term in $P\left(\nu_{\mu} \rightarrow \nu_{e}\right)$}

In this appendix, to see the contribution of the two phases $\delta$ and $\arg \left(\epsilon_{e \tau}\right)$ in $P\left(\nu_{\mu} \rightarrow \nu_{e}\right)$, we will study the $\mathrm{T}$ violating term, which is the last line in Eq. (F1). This term contains the modified Jarlskog factor $\operatorname{Im}\left(\tilde{X}_{1}^{\mu e} \tilde{X}_{2}^{\mu e *}\right)$, and it can be rewritten as

$$
\begin{aligned}
\operatorname{Im}\left(\tilde{X}_{1}^{\mu e} \tilde{X}_{2}^{\mu e *}\right) & =\frac{-1}{\left(\Delta \tilde{E}_{21}\right)^{2} \Delta \tilde{E}_{31} \Delta \tilde{E}_{32}} \operatorname{Im}\left[\left\{Y_{3}^{\mu e}-\left(\tilde{E}_{2}+\tilde{E}_{3}\right) Y_{2}^{\mu e}\right\}\left\{Y_{3}^{\mu e}-\left(\tilde{E}_{3}+\tilde{E}_{1}\right) Y_{2}^{\mu e}\right\}^{*}\right] \\
& =\frac{-1}{\Delta \tilde{E}_{21} \Delta \tilde{E}_{31} \Delta \tilde{E}_{32}} \operatorname{Im}\left\{Y_{3}^{\mu e}\left(Y_{2}^{\mu e}\right)^{*}\right\}
\end{aligned}
$$

In the present case with the ansatz (16), instead of using the explicit expressions (F2), it is convenient to work with the following form:

$$
\begin{aligned}
Y_{2}^{\mu e} & =\left(U \mathcal{E} U^{-1}+\mathcal{A}\right)_{\mu e}=\left(U \mathcal{E} U^{-1}\right)_{\mu e} \\
Y_{3}^{\mu e} & =\left[\left(U \mathcal{E} U^{-1}+\mathcal{A}\right)^{2}\right]_{\mu e}=\left(U \mathcal{E}^{2} U^{-1}\right)_{\mu e}+\left(U \mathcal{E} U^{-1}\right)_{\mu e} \mathcal{A}_{e e}+\left(U \mathcal{E} U^{-1}\right)_{\mu \tau} \mathcal{A}_{\tau e},
\end{aligned}
$$

so that the factor $\operatorname{Im}\left\{Y_{3}^{\mu e}\left(Y_{2}^{\mu e}\right)^{*}\right\}$ in Eq. (G1) can be expressed as

$$
\begin{aligned}
\operatorname{Im}\left\{Y_{3}^{\mu e}\left(Y_{2}^{\mu e}\right)^{*}\right\} & =\operatorname{Im}\left[\left(U \mathcal{E} U^{-1}\right)_{\mu e}^{*}\left\{\left(U \mathcal{E}^{2} U^{-1}\right)_{\mu e}+\left(U \mathcal{E} U^{-1}\right)_{\mu \tau} \mathcal{A}_{\tau e}\right\}\right] \\
& =\operatorname{Im}\left\{Y_{3}^{\mu e}\left(Y_{2}^{\mu e}\right)^{*}\right\}_{\mathrm{std}}+\operatorname{Im}\left\{Y_{3}^{\mu e}\left(Y_{2}^{\mu e}\right)^{*}\right\}_{\mathrm{NP}},
\end{aligned}
$$

where the term $\left(U \mathcal{E} U^{-1}\right)_{\mu e} \mathcal{A}_{e e}$ in $Y_{3}^{\mu e}$ has dropped as in the case of the standard scheme, because $\left|\left(U \mathcal{E} U^{-1}\right)_{\mu e}\right|^{2} \mathcal{A}_{e e}$ is real. In Eq. (G2) we have introduced the notations:

$$
\operatorname{Im}\left\{Y_{3}^{\mu e}\left(Y_{2}^{\mu e}\right)^{*}\right\}_{\text {std }} \equiv \operatorname{Im}\left[\left(U \mathcal{E} U^{-1}\right)_{\mu e}^{*}\left(U \mathcal{E}^{2} U^{-1}\right)_{\mu e}\right]
$$




$$
\begin{aligned}
& =\operatorname{Im}\left[\left\{\left(\Delta E_{31}\right)^{2} X_{3}^{\mu e}+\left(\Delta E_{21}\right)^{2} X_{2}^{\mu e}\right\}\left\{\Delta E_{31}\left(X_{3}^{\mu e}\right)^{*}+\Delta E_{21}\left(X_{2}^{\mu e}\right)^{*}\right\}\right] \\
& =\Delta E_{21} \Delta E_{31} \Delta E_{32} \operatorname{Im}\left\{X_{3}^{\mu e}\left(X_{2}^{\mu e}\right)^{*}\right\}
\end{aligned}
$$

is the Jarlskog factor in the standard three-flavor scheme [74], and

$$
\begin{aligned}
\operatorname{Im}\left\{Y_{3}^{\mu e}\left(Y_{2}^{\mu e}\right)^{*}\right\}_{\mathrm{NP}} \equiv \operatorname{Im}\{( & \left.\left(U \mathcal{E} U^{-1}\right)_{\mu e}^{*}\left(U \mathcal{E} U^{-1}\right)_{\mu \tau} \mathcal{A}_{\tau e}\right\} \\
=\operatorname{Im}[ & \left.A\left(\epsilon_{e \tau}\right)^{*}\left\{\Delta E_{31}\left(X_{3}^{\mu e}\right)^{*}+\Delta E_{21}\left(X_{2}^{\mu e}\right)^{*}\right\}\left(\Delta E_{31} X_{3}^{\mu \tau}+\Delta E_{21} X_{2}^{\mu \tau}\right)\right] \\
=A\left|\epsilon_{e \tau}\right| & {\left[\left(\Delta E_{31}\right)^{2} \operatorname{Im}\left\{\left(X_{3}^{\mu e}\right)^{*} X_{3}^{\mu \tau} e^{2 i \gamma}\right\}\right.} \\
& +\Delta E_{31} \Delta E_{21} \operatorname{Im}\left\{\left(X_{3}^{\mu e}\right)^{*} X_{2}^{\mu \tau} e^{2 i \gamma}+\left(X_{2}^{\mu e}\right)^{*} X_{3}^{\mu \tau} e^{2 i \gamma}\right\} \\
& \left.+\left(\Delta E_{21}\right)^{2} \operatorname{Im}\left\{\left(X_{2}^{\mu e}\right)^{*} X_{2}^{\mu \tau} e^{2 i \gamma}\right\}\right]
\end{aligned}
$$

is the extra contribution to the Jarlskog factor due to new physics. If $\theta_{13}$ is small, then the dominant contribution in the new physics term (G4) comes from the middle one which is proportional to $A\left|\epsilon_{e \tau}\right| \Delta E_{31} \Delta E_{21}\left|X_{2}^{\mu e}\right|\left|X_{3}^{\mu \tau}\right|$, and this should be compared with the standard factor (G3) to examine which contribution dominates the $\mathrm{T}$ violating term.

\section{ACKNOWLEDGMENTS}

The authors thank H. Minakata for useful comments and T. Kajita and K. Kaneyuki for useful communications on the T2K and T2KK experiments. This research was partly supported by a Grant-in-Aid for Scientific Research of the Ministry of Education, Science and Culture, \#21540274, and the MEXT program "Support Program for Improving Graduate School Education."

[1] C. Amsler et al. [Particle Data Group], Phys. Lett. B 667 (2008) 1.

[2] G. L. Fogli, E. Lisi, A. Marrone, A. Palazzo and A. M. Rotunno, Phys. Rev. Lett. 101 (2008) 141801 [arXiv:0806.2649 [hep-ph]].

[3] T. Schwetz, M. A. Tortola and J. W. F. Valle, New J. Phys. 10, 113011 (2008) arXiv:0808.2016v3 [hep-ph]].

[4] G. L. Fogli, E. Lisi, A. Marrone, A. Palazzo and A. M. Rotunno, arXiv:0809.2936 [hep-ph].

[5] H. L. Ge, C. Giunti and Q. Y. Liu, arXiv:0810.5443 [hep-ph].

[6] G. L. Fogli, E. Lisi, A. Marrone, A. Palazzo and A. M. Rotunno, arXiv:0905.3549 [hep-ph].

[7] M. C. Gonzalez-Garcia, M. Maltoni and J. Salvado, arXiv:1001.4524 [hep-ph].

[8] A. Bandyopadhyay et al. [ISS Physics Working Group], Rept. Prog. Phys. 72, 106201 (2009) arXiv:0710.4947 [hep-ph]].

[9] Belle experiment, http://belle.kek.jp/.

[10] Babar experiment, http://www-public.slac.stanford.edu/babar/.

[11] L. Wolfenstein, Phys. Rev. D 17, 2369 (1978).

[12] M. M. Guzzo, A. Masiero and S. T. Petcov, Phys. Lett. B 260 (1991) 154.

[13] E. Roulet, Phys. Rev. D 44 (1991) 935.

[14] S. P. Mikheev and A. Y. Smirnov, Sov. J. Nucl. Phys. 42, 913 (1985) [Yad. Fiz. 42, 1441 (1985)]. 
[15] S. Davidson, C. Pena-Garay, N. Rius and A. Santamaria, JHEP 0303, 011 (2003) arXiv:hep-ph/0302093.

[16] N. Fornengo, M. Maltoni, R. Tomas and J. W. F. Valle, Phys. Rev. D 65, 013010 (2002) arXiv:hep-ph/0108043.

[17] Z. Berezhiani and A. Rossi, Phys. Lett. B 535 (2002) 207 arXiv:hep-ph/0111137.

[18] M. C. Gonzalez-Garcia and M. Maltoni, Phys. Rev. D 70, 033010 (2004) arXiv:hep-ph/0404085.

[19] O. G. Miranda, M. A. Tortola and J. W. F. Valle, JHEP 0610, 008 (2006) arXiv:hep-ph/0406280.

[20] J. Barranco, O. G. Miranda, C. A. Moura and J. W. F. Valle, Phys. Rev. D 73 (2006) 113001 arXiv:hep-ph/0512195.

[21] J. Barranco, O. G. Miranda, C. A. Moura and J. W. F. Valle, Phys. Rev. D 77 (2008) 093014 arXiv:0711.0698 [hep-ph]].

[22] A. Bolanos, O. G. Miranda, A. Palazzo, M. A. Tortola and J. W. F. Valle, Phys. Rev. D 79, 113012 (2009) arXiv:0812.4417 [hep-ph]].

[23] F. J. Escrihuela, O. G. Miranda, M. A. Tortola and J. W. F. Valle, Phys. Rev. D 80, 105009 (2009) [Erratum-ibid. D 80, 129908 (2009)] [arXiv:0907.2630 [hep-ph]].

[24] C. Biggio, M. Blennow and E. Fernandez-Martinez, JHEP 0908, 090 (2009) arXiv:0907.0097 [hep-ph]].

[25] C. Biggio, M. Blennow and E. Fernandez-Martinez, JHEP 0903, 139 (2009) arXiv:0902.0607 [hep-ph]].

[26] N. Kitazawa, H. Sugiyama and O. Yasuda, arXiv:hep-ph/0606013.

[27] S. Davidson, private communications (2008).

[28] O. Yasuda, PoS NUFACT2008, 016 (2008).

[29] A. Friedland, C. Lunardini and M. Maltoni, Phys. Rev. D 70, 111301 (2004) arXiv:hep-ph/0408264.

[30] A. Friedland and C. Lunardini, Phys. Rev. D 72 (2005) 053009 arXiv:hep-ph/0506143.

[31] A. Friedland, C. Lunardini and C. Pena-Garay, Phys. Lett. B 594 (2004) 347 arXiv:hep-ph/0402266.

[32] A. Palazzo and J. W. F. Valle, Phys. Rev. D 80 (2009) 091301 arXiv:0909.1535 [hep-ph]].

[33] MINOS experiment, http://www-numi.fnal.gov/.

[34] A. Friedland and C. Lunardini, Phys. Rev. D 74, 033012 (2006) arXiv:hep-ph/0606101.

[35] O. Yasuda, Acta Phys. Polon. B 38, 3381 (2007) arXiv:0710.2601 [hep-ph]].

[36] H. Sugiyama, AIP Conf. Proc. 981, 216 (2008) [arXiv:0711.4303 [hep-ph]].

[37] M. Blennow, T. Ohlsson and J. Skrotzki, Phys. Lett. B 660, 522 (2008) arXiv:hep-ph/0702059v3].

[38] OPERA experiment, http://operaweb.lngs.infn.it/.

[39] A. Esteban-Pretel, J. W. F. Valle and P. Huber, Phys. Lett. B 668 (2008) 197 arXiv:0803.1790 [hep-ph]].

[40] M. Blennow, D. Meloni, T. Ohlsson, F. Terranova and M. Westerberg, Eur. Phys. J. C 56 (2008) 529 arXiv:0804.2744 [hep-ph]].

[41] N. C. Ribeiro, H. Nunokawa, T. Kajita, S. Nakayama, P. Ko and H. Minakata, Phys. Rev. D 77, 073007 (2008) arXiv:0712.4314 [hep-ph]].

[42] M. Ishitsuka, T. Kajita, H. Minakata and H. Nunokawa, Phys. Rev. D 72, 033003 (2005) arXiv:hep-ph/0504026. 
[43] K. Hagiwara, N. Okamura and K. i. Senda, Phys. Lett. B 637, 266 (2006) [Erratum-ibid. B 641, 486 (2006)] arXiv:hep-ph/0504061].

[44] Y. Itow et al. [The T2K Collaboration], arXiv:hep-ex/0106019.

[45] J. Kopp, M. Lindner, T. Ota and J. Sato, Phys. Rev. D 77 (2008) 013007 arXiv:0708.0152 [hep-ph]].

[46] S. Geer, Phys. Rev. D 57, 6989 (1998) [Erratum-ibid. D 59, 039903 (1999)] arXiv:hep-ph/9712290.

[47] A. M. Gago, M. M. Guzzo, H. Nunokawa, W. J. C. Teves and R. Zukanovich Funchal, Phys. Rev. D 64 (2001) 073003 arXiv:hep-ph/0105196.

[48] T. Ota, J. Sato and N. a. Yamashita, Phys. Rev. D 65 (2002) 093015 arXiv:hep-ph/0112329.

[49] P. Huber, T. Schwetz and J. W. F. Valle, Phys. Rev. Lett. 88 (2002) 101804 arXiv:hep-ph/0111224.

[50] M. Campanelli and A. Romanino, Phys. Rev. D 66 (2002) 113001 [arXiv:hep-ph/0207350].

[51] N. C. Ribeiro, H. Minakata, H. Nunokawa, S. Uchinami and R. Zukanovich-Funchal, JHEP 0712 (2007) 002 [arXiv:0709.1980 [hep-ph]].

[52] J. Kopp, T. Ota and W. Winter, Phys. Rev. D 78 (2008) 053007 [arXiv:0804.2261 [hep-ph]].

[53] A. M. Gago, H. Minakata, H. Nunokawa, S. Uchinami and R. Zukanovich Funchal, JHEP 1001, 049 (2010) arXiv:0904.3360 [hep-ph]].

[54] D. Meloni, T. Ohlsson, W. Winter and H. Zhang, arXiv:0912.2735 [hep-ph].

[55] J. Burguet-Castell, M. B. Gavela, J. J. Gomez-Cadenas, P. Hernandez and O. Mena, Nucl. Phys. B 608, 301 (2001) arXiv:hep-ph/0103258.

[56] H. Minakata and H. Nunokawa, JHEP 0110, 001 (2001) arXiv:hep-ph/0108085].

[57] G. L. Fogli and E. Lisi, Phys. Rev. D 54 (1996) 3667 arXiv:hep-ph/9604415;

[58] V. Barger, D. Marfatia and K. Whisnant, Phys. Rev. D 65, 073023 (2002) arXiv:hep-ph/0112119.

[59] G. Mitsuka [Super-Kamiokande Collaboration], PoS NUFACT08, 059 (2008).

[60] J. Hosaka et al. [Super-Kamiokande Collaboration], Phys. Rev. D 74, 032002 (2006) arXiv:hep-ex/0604011.

[61] K. Hagiwara, N. Okamura and K. i. Senda, Phys. Rev. D 76, 093002 (2007) arXiv:hep-ph/0607255.

[62] T. Kajita, H. Minakata, S. Nakayama and H. Nunokawa, Phys. Rev. D 75, 013006 (2007) arXiv:hep-ph/0609286.

[63] K. Hagiwara and N. Okamura, JHEP 0801, 022 (2008) arXiv:hep-ph/0611058.

[64] K. Hagiwara and N. Okamura, JHEP 0907, 031 (2009) arXiv:0901.1517 [hep-ph]].

[65] K. Kaneyuki, talk at 4th International Workshop on Nuclear and Particle Physics at J-PARC (NP08), Mito, Ibaraki, Japan , 5-7 Mar 2008, http://nuclpart.kek.jp/NP08/presentations/neutrino/pdf/NP08_kaneyuki.pdf.

[66] A. Rubbia and A. Meregaglia, Talk at the 2nd International Workshop on a Far Detector in Korea for the J-PARC Neutrino Beam, Seoul National University, Seoul, July 13-14, 2006, http://www-rccn.icrr.u-tokyo.ac.jp/workshop/T2KK06/files/06_JPARC_beam_profile_T2KK.pdf

[67] O. Yasuda, arXiv:0704.1531 [hep-ph].

[68] K. Kimura, A. Takamura and H. Yokomakura, Phys. Lett. B 537, 86 (2002) arXiv:hep-ph/0203099.

[69] K. Kimura, A. Takamura and H. Yokomakura, Phys. Rev. D 66, 073005 (2002) arXiv:hep-ph/0205295.

[70] Z. z. Xing and H. Zhang, Phys. Lett. B 618, 131 (2005) arXiv:hep-ph/0503118. 
[71] D. Meloni, T. Ohlsson and H. Zhang, JHEP 0904, 033 (2009) arXiv:0901.1784 [hep-ph]].

[72] O. Yasuda, Proceedings of Symposium on New Era in Neutrino Physics (Universal Academy Press, Inc., Tokyo, eds. H. Minakata and O. Yasuda), p 165 - 177 (1999) arXiv:hep-ph/9809205.

[73] Y. Ashie et al. [Super-Kamiokande Collaboration], Phys. Rev. D 71, 112005 (2005) arXiv:hep-ex/0501064.

[74] V. A. Naumov, Int. J. Mod. Phys. D 1, 379 (1992). 